\title{
ERWANN DELAY
}

\section{Analyse précisée d'équations semi-linéaires elliptiques sur l'espace hyperbolique et application à la courbure scalaire conforme}

Bulletin de la S. M. F., tome 125, no 3 (1997), p. 345-381

<http://www.numdam.org/item?id=BSMF_1997_125_3_345_0>

(C) Bulletin de la S. M. F., 1997, tous droits réservés.

L'accès aux archives de la revue "Bulletin de la S. M. F. » (http: //smf.emath.fr/Publications/Bulletin/Presentation.html) implique l'accord avec les conditions générales d'utilisation (http://www.numdam.org/ conditions). Toute utilisation commerciale ou impression systématique est constitutive d'une infraction pénale. Toute copie ou impression de ce fichier doit contenir la présente mention de copyright.

\section{Numdam}




\title{
ANALYSE PRÉCISÉE D'ÉQUATIONS SEMI-LINÉAIRES ELLIPTIQUES SUR L'ESPACE HYPERBOLIQUE ET APPLICATION À LA COURBURE SCALAIRE CONFORME
}

PAR

\author{
ERWANN DELAY (*)
}

\begin{abstract}
RÉSUMÉ. - Au thème de la courbure scalaire conforme sur l'espace hyperbolique nous apportons ici une étude fine du comportement asymptotique en toute dimension. Nous traitons toujours d'équations semi-linéaires générales, avant d'appliquer nos résultats au cas particulier de l'équation géométrique.

ABSTRACT. - Regarding the theme of the conformal scalar curvature on the hyperbolic space, we bring here a study of the fine asymptotic behavior in any dimension. We always deal with general semi-linear equations, before applying our results to the particular case of the geometric equation.
\end{abstract}

\section{Introduction}

Soit $B$ la boule unitée de $\mathbb{R}^{n}$ (munie de la métrique euclidienne standard $E$ ) et soit $\rho$ la fonction définie sur $B$ par :

$$
\rho(x)=\frac{1}{2}\left(1-|x|^{2}\right) \text {. }
$$

Nous utiliserons pour modèle de l'espace hyperbolique standard $\mathbb{H}^{n}(-1)$ la boule $B$ munie de la métrique conforme :

$$
H_{0}=\rho^{-2} E \text {. }
$$

(*) Texte reçu le 18 décembre 1996, révisé le 4 mars 1997, accepté le 22 avril 1997.

E. Delay, Moniteur-Allocataire menesr, Université de Nice-Sophia Antipolis, Mathématiques, Parc Valrose, B.P. 71, 06108 Nice CEDEX 2 (France).

Email : delay@math.unice.fr

Mots clés : espace hyperbolique, métriques conformes, courbure scalaire, EDP semilinéaires elliptiques, estimations a priori, comportement asymptotique, existence, inexistence, sur et sous solutions, méthode de continuité.

Classification AMS : 58 G 30, 53 C 21, 35 J 70, 35 B 45, 35 B 40. 
$\mathrm{Au}$ départ de cet article on trouve le fait suivant : l'application qui, à une fonction réelle $v$ définie sur $B$, associe la courbure scalaire de la métrique conforme $H_{v}=(1+v) H_{0}$, est localement inversible au voisinage de $v=0$, lorsqu'on la considère dans des espaces fonctionnels appropriés. Les espaces en question, utilisés dans [GL], dépendent d'un paramètre réel $s$ caractéristique du comportement à l'infini des fonctions et de leurs dérivées. Rappelons-en la définition : une fonction $u \in \mathrm{C}_{\mathrm{loc}}^{k, \alpha}$ est dans l'espace $\Lambda_{k, \alpha}^{s}$ si la quantité suivante, qui représente sa norme dans cet espace, est finie :

$$
\begin{aligned}
\|u\|_{k, \alpha}^{s} & :=\sum_{|\gamma| \leq k} \sup _{x \in B}\left[d_{x}^{-s+|\gamma|}\left|\partial^{\gamma} u(x)\right|\right] \\
& +\sum_{|\gamma|=k} \sup _{\substack{x, y \in B \\
x \neq y}} \min \left(d_{x}^{-s+k+\alpha}, d_{y}^{-s+k+\alpha}\right) \frac{\left|\partial^{\gamma} u(x)-\partial^{\gamma} u(y)\right|}{|x-y|^{\alpha}}
\end{aligned}
$$

où $d_{x}$ est la distance euclidienne de $x$ au bord (en l'occurence $d_{x} \equiv 1-|x|$ ). Un membre typique de $\Lambda^{s}$ est la fonction $\rho^{s}$.

Si $\Delta$ désigne le laplacien de la métrique $H_{0}$ (dont le symbole appliqué à un covecteur $\xi$ est par convention, égal à $\left.-|\xi|_{H_{0}}^{2}\right)$, et si $K \in \Lambda_{k, \alpha}^{0}$ vérifie la minoration stricte suivante

$$
\inf _{B} K>s[s-(n-1)]
$$

alors l'opérateur de type Schrödinger

$$
\Delta+K: \Lambda_{k+2, \alpha}^{s} \longrightarrow \Lambda_{k, \alpha}^{s}
$$

est un isomorphisme. Ce résultat essentiel de [GL, p. 217], formulé avec $K \in C^{\infty}(\bar{B})$ mais encore vrai avec $K \in \Lambda_{k, \alpha}^{0}$, nous sera d'un usage constant; nous l'invoquerons ci-après sous le vocable de «théorème d'isomorphisme de $[\mathrm{GL}] »$. C'est lui qui intervient $(\S 2)$ pour démontrer que l'application :

$$
v \in \Lambda_{k+2, \alpha}^{s} \longrightarrow \operatorname{Scal}\left(H_{v}\right)-\operatorname{Scal}\left(H_{0}\right) \in \Lambda_{k, \alpha}^{s}
$$

est localement inversible près de $v=0$ dès que $s$ appartient à $[0, n[$. Voilà donc précisé ce fait de départ.

Dans le paragraphe 2, nous montrerons que ce fait vient s'imbriquer parfaitement à côté d'un important théorème de rigidité de Min-Oo [MO1] dont il a catalysé une rectification d'hypothèse [MO2]. Selon ce théorème

TOME $125-1997-\mathrm{N}^{\circ} 3$ 
rectifié, dans le cas particulier de la boule $B$, si une métrique $H$ est convenablement asymptotique à $H_{0}$ à l'infini (pour nous $H_{v}$ le sera précisément dès que $s>n$ ), alors il est impossible d'avoir $\operatorname{Scal}(H) \geq \operatorname{Scal}\left(H_{0}\right)$ sauf si $H$ est isométrique à $H_{0}$, auquel cas $\operatorname{Scal}(H)=\operatorname{Scal}\left(H_{0}\right)$. D'après l'inversion locale indiquée plus haut, ce résultat ne tient plus dès lors que $s$ appartient à $[0, n[$. Ce théorème peut être vu comme un analogue, en asymptotiquement hyperbolique (où l'on ne dispose toutefois pas encore d'une notion convenable de masse), du théorème de la masse positive en asymptotiquement euclidien (voir e.g. le paragraphe 3 de Leung [L]).

Finalement, ce fait de départ nous pousse à étudier de façon plus globale l'application «courbure scalaire conforme», considérée dans les espaces $\Lambda^{s}$, en dimension $n>2(\S 3)$ et $n=2(\S 4)$, sur $B$ munie de la métrique hyperbolique standard $H_{0}$. Une telle étude vient compléter les travaux antérieurs sur le sujet [AM], [CCY], [LTY], [RRV] dans lesquels le comportement au voisinage de l'infini n'est précisé qu'à l'ordre zéro. Or c'est l'étude du comportement à l'infini qui a permis de tester la validité du théorème de Min-Oo [MO2]. Au paragraphe 5, nous construisons un exemple qui met directement en évidence les limites de notre méthode lorsque la valeur du paramètre de pondération $s$ est située hors de l'intervalle prescrit par le théorème d'isomorphisme de [GL].

Nous avons regroupé en appendice $(\S 6)$ divers compléments techniques absents de [GL].

Indiquons enfin que l'analyse dans les espaces $\Lambda^{s}$ nous permet aussi d'étudier, dans un autre travail en cours, la prescriptibilité locale de la coubure de Ricci au voisinage de la métrique hyperbolique (sans plus se restreindre à la classe conforme).

Mes remerciements vont à Philippe Delanoë, qui encadre ma thèse à l'Université de Nice, où je suis allocataire de recherche du MEnESR depuis octobre 1994. Je remercie aussi Maung Min-Oo pour son accueil constructif d'une version préliminaire de ce travail, ainsi que Marc Herzlich et le referee de cet article, pour leurs commentaires concernant la masse. Enfin je remercie Gilles Laschon et François Gautero pour leurs remarques et leur amical soutien.

\section{Prescriptibilité locale de la courbure scalaire conforme}

La courbure scalaire d'une variété Riemannienne munie d'une métrique $H$ est donnée par :

$$
\operatorname{Scal}(H)=\operatorname{Trace}_{H}(R) \quad \text { où } \quad R=\operatorname{Ricci}(H) .
$$

La linéarisation de $H \mapsto \operatorname{Scal}(H)$ est donnée par (cf. e.g. [B, p. 63])

$$
\mathrm{d}(\operatorname{Scal})(H) \delta H=\Delta \tau+\nabla^{i j}(\delta H)_{i j}-R^{i j}(\delta H)_{i j}
$$


où :

- $\tau=\operatorname{Trace}_{H} \delta H$

- $\nabla$ désigne la connexion de Levi-Civita de $H$ et

- $\Delta=-\operatorname{Trace}_{H}\left(\nabla^{2}\right)$ son laplacien.

Si $H$ est d'Einstein, on a :

$$
R^{i j}=\frac{1}{n} \operatorname{Scal}(H) H^{i j}
$$

Dans ce cas :

$$
\mathrm{d}(\operatorname{Scal})(H) \delta H=\Delta \tau+\nabla^{i j}(\delta H)_{i j}-\frac{1}{n} \operatorname{Scal}(H) \tau
$$

et lorsque $\operatorname{Scal}(H)=-n(n-1)$, comme en $H_{0}$ (et en toutes les métriques d'Einstein construites par [GL]) :

$$
\mathrm{d}(\operatorname{Scal})(H) \delta H=\Delta \tau+\nabla^{i j}(\delta H)_{i j}+(n-1) \tau .
$$

REMARQUe. - L'opérateur différentiel

$$
\delta H \longmapsto \Delta \tau+\nabla^{i j}(\delta H)_{i j}+(n-1) \tau
$$

est elliptique sous-déterminé (i.e. son symbole est surjectif). En effet son terme principal s'écrit localement :

$$
\left(H^{i k} H^{j \ell}-H^{i j} H^{k \ell}\right) \partial_{i j}(\delta H)_{k \ell} .
$$

Pour tout covecteur $\xi \neq 0$ :

$$
\left(H^{i k} H^{j \ell}-H^{i j} H^{k \ell}\right) \xi_{i} \xi_{j}=\left(\xi^{k} \xi^{\ell}-|\xi|^{2} H^{k \ell}\right),
$$

et $L: \delta H \mapsto\left(\xi^{k} \xi^{\ell}-|\xi|^{2} H^{k \ell}\right)(\delta H)_{k \ell}$ est une application linéaire surjective. En effet, pour tout $\eta \in \mathbb{R}$, on a :

$$
L\left(\frac{-\eta}{(n-1)|\xi|^{2}} H\right)=\eta
$$

Cette démonstration suggère de se restreindre à des variations conformes de $H$, i.e. de prendre $\delta H=\frac{\tau}{n} H$ (ainsi $\tau=\operatorname{Trace}_{H} \delta H$ ) pour laquelle

$$
\nabla^{i j}(\delta H)_{i j}=-\frac{\Delta \tau}{n} \quad \text { et } \quad \mathrm{d}(\operatorname{Scal})(H)(\delta H)=\frac{n-1}{n}(\Delta \tau+n \tau) .
$$

D'après [GL], si $H=H_{0}$, alors $\Delta+n$ est un isomorphisme de $\Lambda_{k+2, \alpha}^{s}$ dans $\Lambda_{k, \alpha}^{s}$ dès que $n>s(s-(n-1))$, i.e. dès que $-1<s<n$. Ainsi en vertu du théorème d'inversion locale on obtient le : 
ThÉorème 1. - Soient $k \in \mathbb{N}, \alpha \in] 0,1[$ et $s \in[0, n[$. Pour toute fonction $\sigma$ assez proche de zéro dans $\Lambda_{k, \alpha}^{s}$, il existe une unique fonction $v$ assez proche de zéro dans $\Lambda_{k+2, \alpha}^{s}$, dépendant différentiablement de $\sigma$ telle que la métrique $H_{v}=(1+v) H_{0}$ vérifie

$$
\operatorname{Scal}\left(H_{v}\right)=\operatorname{Scal}\left(H_{0}\right)+\sigma=-n(n-1)+\sigma .
$$

Il n'est pas difficile d'établir qu'il existe au plus une solution $v$ de l'équation précédente dès lors que $s>0$ et $\operatorname{Scal}\left(H_{0}\right)+\sigma \leq 0$ (cela découle du principe du maximum). Par contre, l'existence d'une solution $v$ dans le bon espace, lorsque $s \geq n$, n'est plus assurée : nous construisons un exemple le démontrant directement pour $s>n+1$ au paragraphe 5 ciaprès. Pour lors, à ce sujet nous allons procéder indirectement, par une comparaison entre le théorème 1 et un important résultat de rigidité dû à Min-Oo [MO1], [MO2] (voir aussi [L]).

En prenant comme modèle de l'espace hyperbolique $\mathbb{H}^{n}, \mathbb{R}^{n}$ muni de la métrique qui s'exprime en coordonnées polaires à l'origine par

$$
\bar{H}_{0}=\mathrm{d} R^{2}+(\operatorname{sh} R)^{2} \mathrm{~d} \Theta^{2}
$$

où $R \in] 0, \infty\left[\right.$ et $\mathrm{d} \Theta^{2}$ désigne la métrique standard de $S^{n-1}$.

Rappelons (voir [MO1], [MO2]) la :

DÉfinition 1. - Une variété Riemannienne $(\mathrm{M}, \mathrm{H})$ est dite fortement asymptotiquement hyperbolique s'il existe un compact $K$ de $M$ et un difféomorphisme

$$
\Phi: M \backslash K \longrightarrow \mathbb{R}^{n} \backslash \overline{B_{R_{0}}(0)}
$$

pour un $R_{0} \geq 0$ tel que si on définit la transformation de jauge

$$
A: T(M \backslash K) \longrightarrow T(M \backslash K)
$$

par :

(i) $H(A u, A v)=\left(\Phi^{\star} \overline{H_{0}}\right)(u, v)$;

(ii) $H(A u, v)=H(u, A v)$;

alors $A$ vérifie :

$\left(\mathrm{AH}_{1}\right)$ : il existe $C \geq 1$ tel que, pour tout $v \in T(M \backslash K)$,

$$
C^{-1} H(v, v) \leq H(A v, A v) \leq C H(v, v) ;
$$

$\left(\mathrm{AH}_{2}\right)$ : le champ d'endomorphismes sur $M \backslash K$ défini par

$$
x \in M \backslash K \longmapsto \mathrm{e}^{|\Phi(x)|}(A-\mathrm{Id})(x) \in T^{\star}(M \backslash K) \otimes T(M \backslash K)
$$


(où $|\cdot|$ désigne la norme euclidienne standard de $\mathbb{R}^{n}$ ) est dans l'intersection des espaces de Sobolev $H_{1}^{2} \cap H_{1}^{1}$ sur $M \backslash K$ relativement à $H$.

Nota Bene. - Par continuité à partir d'un voisinage de l'infini, on voit que les valeurs propres de $A$ sont nécessairement positives sur $M \backslash K$ (elles le sont à l'infini et ne peuvent s'annuler à cause de (i), a fortiori à cause de $\mathrm{AH}_{1}$ ).

Le résultat visé par Min-Oo s'énonce alors :

ThÉORÈme 2. - Si $(M, H)$ est spinorielle fortement asymptotiquement hyperbolique de dimension $n>2$ à courbure scalaire $S \geq S_{0}=\operatorname{Scal}\left(H_{0}\right)$, alors $(M, H)$ est isométrique à $\mathbb{H}^{n}$ (donc $\left.S=S_{0}\right)$.

Adaptons cette définition et ce théorème au cas particulier où

$$
M=B:=\left\{x \in \mathbb{R}^{n} ;|x|<1\right\}
$$

munie de $H$, qui est évidemment spinorielle $\left(w_{2}(B)=0\right)$, quand on prend plutôt pour modèle de $\mathbb{H}^{n}, B$ munie de $H_{0}=\rho^{-2} E, E$ étant la métrique euclidienne standard et $\rho$ la fonction définie par

$$
\rho(x)=\frac{1}{2}\left(1-|x|^{2}\right)
$$

(ici encore, $|\cdot|$ désigne la norme euclidienne standard de $\mathbb{R}^{n}$ ).

Considérons $\Phi: B \backslash\{0\} \rightarrow \mathbb{R}^{n} \backslash\{0\}$ définie en coordonnées polaires à l'origine par $(r, \Theta) \mapsto(R=2 \operatorname{argth}(r), \Theta)$; alors $\Phi^{\star} \bar{H}_{0}=H_{0}$. Sur $B$, la transformation de jauge $A$ de la définition 1 est définie par :

$$
\begin{aligned}
A_{i}^{\ell} A_{j}^{k} H_{k \ell} & =H_{0 i j}, \\
A_{i}^{k} H_{k \ell} & =A_{\ell}^{k} H_{k i} .
\end{aligned}
$$

Si l'on prend $H$ sous la forme $H_{v}=(1+v) H_{0}$ pour $v \in \Lambda_{k+2, \alpha}^{s}$ (voir la définition $\S 1$ ) et $\|v\|_{k+2, \alpha}^{s}<1$, que devient la condition $\mathrm{AH}_{2}$ (ci-dessus)?

REMARQUes.

(i) On a déjà $A=(1+u)$ Id avec $u \in \Lambda_{k+2, \alpha}^{s}$ et $(1+u)^{2}(1+v)=1$.

(ii) On a :

$$
\begin{aligned}
\sqrt{\operatorname{det} H} & =\sqrt{\operatorname{det}\left((1+v) H_{0}\right)}=(1+v)^{\frac{1}{2} n} \sqrt{\operatorname{det} H_{0}} \\
& =(1+v)^{\frac{1}{2} n} \rho^{-n}=\frac{1}{(1+u)^{n}} \rho^{-n} \in \Lambda_{k+2, \alpha}^{-n} .
\end{aligned}
$$

TOME $125-1997-\mathrm{N}^{\circ} 3$ 
(iii) On a

$$
\mathrm{e}^{|\Phi(x)|}=\mathrm{e}^{\ln \left(\frac{1+|x|}{1-|x|}\right)}=\frac{1+|x|}{1-|x|} \in \Lambda_{\infty}^{-1}
$$

(iv) On a

$$
\mathrm{e}^{|\Phi|}(A-\mathrm{Id})=\mathrm{e}^{|\Phi|} u \operatorname{Id} \in \Lambda_{k+2, \alpha}^{s-1}
$$

en tant que tenseur de type $\left(\begin{array}{l}1 \\ 1\end{array}\right)$ et donc

$$
\nabla\left[\mathrm{e}^{|\Phi|}(A-\mathrm{Id})\right] \in \Lambda_{k+1, \alpha}^{s-2}
$$

en tant que tenseur de type $\left(\begin{array}{l}1 \\ 2\end{array}\right)$.

Comme la condition $\mathrm{AH}_{2}$ s'écrit :

$$
\int_{B}\left\|\mathrm{e}^{|\Phi|} u \mathrm{Id}\right\|_{H}^{2} \sqrt{\operatorname{det} H} \mathrm{~d} x<\infty, \quad \int_{B}\left\|\nabla\left(\mathrm{e}^{|\Phi|} u \mathrm{Id}\right)\right\|_{H}^{2} \sqrt{\operatorname{det} H} \mathrm{~d} x<\infty,
$$

et

$$
\int_{B}\left\|\mathrm{e}^{|\Phi|} u \operatorname{Id}\right\|_{H} \sqrt{\operatorname{det} H} \mathrm{~d} x<\infty, \quad \int_{B}\left\|\nabla\left(\mathrm{e}^{|\Phi|} u \mathrm{Id}\right)\right\|_{H} \sqrt{\operatorname{det} H} \mathrm{~d} x<\infty
$$

et que pour $a \in T^{\star}(B) \otimes T(B)$, on a

$$
\|a\|_{H}^{2}=H^{\ell k} a_{\ell}^{i} a_{k}^{j} H_{i j} \in \Lambda^{2 s-2} \quad \text { si } \quad a \in \Lambda^{s-1}
$$

et pour $b \in T^{\star}(B) \otimes T^{\star}(B) \otimes T(B)$,

$$
\|b\|_{H}^{2}=H^{j k} H^{\ell p} b_{j \ell}^{i} b_{p q}^{k} H_{i k} \in \Lambda^{2 s-2} \quad \text { si } \quad b \in \Lambda^{s-2},
$$

alors, compte-tenu des remarques (ii) et (iv) précédentes :

$$
\begin{aligned}
\mathrm{AH}_{2} & \Longleftrightarrow \int_{B} \rho^{s-1-n} \mathrm{~d} x<\infty \\
& \Longleftrightarrow \int_{B}\left(1-|x|^{2}\right)^{s-1-n} \mathrm{~d} x<\infty \\
& \Longleftrightarrow s>n .
\end{aligned}
$$

On remarque de plus que $\mathrm{AH}_{1}$ est vérifiée dès que $|u|$ est assez petite (i.e. $|v|$ assez petite). Ainsi il existe $\epsilon>0$ tel que, si $\|v\|_{k+2, \alpha}^{s}<\epsilon$ et si $s>n$, la variété $B$ munie de la métrique Riemannienne $H_{v}=(1+v) H_{0}$ est fortement asymptotiquement hyperbolique. Pour de telles métriques, le théorème 2 et un argument d'unicité conduisent au : 
Corollaire 1. - Soient $k \in \mathbb{N}, \alpha \in] 0,1\left[, s>n\right.$ et $v \in \Lambda_{k+2, \alpha}^{s}$ avec $\|v\|_{k+2, \alpha}^{s}$ assez petite. Si $\operatorname{Scal}\left(H_{v}\right) \geq \operatorname{Scal}\left(H_{0}\right)$, alors $v \equiv 0$.

Notons que d'après le théorème 1 , pour tout $\sigma \in \Lambda_{k, \alpha}^{s}$ non négatif assez petit et tout $t \in\left[0, n\right.$ [, compte-tenu de l'inclusion $\Lambda_{k, \alpha}^{s} \subset \Lambda_{k, \alpha}^{t}$ (voir [GL]), il existe une unique solution $v \in \Lambda_{k+2, \alpha}^{t}$ de l'équation

$$
\operatorname{Scal}\left(H_{v}\right)=\operatorname{Scal}\left(H_{0}\right)+\sigma .
$$

Bien sûr, $\sigma \not \equiv 0$ implique $v \not \equiv 0$. Le corollaire 1 implique que cette solution n'est pas dans $\Lambda_{k+2, \alpha}^{s}$. Ainsi les théorèmes 1 et 2 se complètent-ils exactement de part et d'autre de l'exposant critique $s=n$.

Dans la suite de cet article, motivés par l'inversibilité locale (en $v=0$ ) de la courbure scalaire conforme considérée dans les espaces $\Lambda^{s}$ de [GL], nous allons, par des techniques d'analyse non-linéaire appropriées, passer du résultat purement local précédent à des résultats globaux, en précisant en particulier «la taille» dans $\Lambda_{k, \alpha}^{s}$ des $\sigma$ pour lesquels il existe une solution $v \in \Lambda_{k+2, \alpha}^{s}$ de l'équation $\operatorname{Scal}\left[(1+v) H_{0}\right]=S_{0}+\sigma$.

\section{Courbure scalaire conforme en dimension supérieure à 2}

Dans cette partie, on se propose d'abord d'étudier dans la boule $B$ munie de la métrique $H_{0}$, l'équation :

$$
\Delta v=(A-f)(1+v)+(g-A)(1+v)^{p} \quad \text { avec } \quad v>-1,
$$

où $A$ et $p$ sont réels, $A>0, p>1, f$ et $g$ sont des fonctions données sur $B$, avec $g \leq A$. Il sera parfois commode d'abréger le second membre de $(\mathrm{E})$ en le notant $\Phi(x, v)$. Nous allons tour à tour démontrer, sous des conditions appropriées, l'existence d'une solution de (E), puis son unicité, enfin sa régularité. Nous appliquerons ensuite cette étude à l'équation, cas particulier de (E), qui traduit en dimension $n>2$ la prescription sur $B$ de la courbure scalaire d'une métrique conforme à $\mathrm{E}$ et asymptotique à $H_{0}$ à l'infini (près du bord de $B$ ).

\section{1. Étude EDP (dimension $n$ quelconque).}

3.1.1 Existence. - Réécrivons l'équation (E) sous la forme :

$$
\begin{aligned}
\Delta v+A(p-1) v & =(A-f)(1+v)+(g-A)(1+v)^{p}+A(p-1) v \\
& =: \widetilde{\Phi}(x, v) .
\end{aligned}
$$

Soit $M=M(n, s, A(p-1))$ la constante de l'appendice 6.3 (ci-après, telle que $\varphi=M \rho^{s}$ vérifie $\left.[\Delta+A(p-1)] \varphi \geq \rho^{s}\right)$. Dans tout ce qui suit, chaque fois qu'on utilisera la condition (C) de l'appendice 6.3 , on l'entendra lue avec $K=A(p-1)$. 
Proposition 1. - Sif et $g$ sont dans $\Lambda_{0}^{s} \cap \mathrm{C}_{\mathrm{loc}}^{k, \alpha}, s \geq 0$ vérifiant $(\mathrm{C})$, $0<\alpha<1$ et s'il existe des réels $\lambda \geq 0$ et $\delta \in] 0,1[$ tels que, en posant $q=\frac{1-\delta^{p}}{1-\delta}$ (donc $q$ est dans $] 1, p[$ ), on ait l'encadrement :

$$
\begin{aligned}
-(1-\delta)\left[\frac{1}{M}-A(p-1)\right. & +q(A-g)+f-A] \\
\leq & (2 \rho)^{-s}(g-f) \leq \lambda\left(\frac{1}{M}+f-p g\right)
\end{aligned}
$$

alors il existe une solution $v>-1$ de $(\mathrm{E})$ dans $\Lambda_{0}^{s} \cap C_{\mathrm{loc}}^{k+2, \alpha}$.

Preuve. - Nous allons résoudre (E) en construisant pour elle des solutions supérieure et inférieure, i.e. en construisant $v^{+} \geq v^{-}>-1$ telles que $\Delta v^{+} \geq \Phi\left(x, v^{+}\right)$et $\Delta v^{-} \leq \Phi\left(x, v^{-}\right)$. D'après une procédure bien connue (voir e.g. $[\mathrm{N}]$ ), l'existence d'une solution $v>-1$ comprise entre $v^{+}$et $v^{-}$en résulte.

- Soit

$$
v^{+}=\frac{2^{s} \lambda}{M} \varphi=\lambda(2 \rho)^{s} .
$$

Alors $0<v^{+} \leq \lambda$ et comme $t \mapsto(1+t)^{p}$ est convexe pour $p>1$, on a $(1+t)^{p} \geq 1+p t$ pour tout $t \geq 0$; ainsi :

$$
\begin{aligned}
\widetilde{\Phi}\left(x, v^{+}\right) & \leq(A-f)\left(1+v^{+}\right)+(g-A)\left(1+p v^{+}\right)+A(p-1) v^{+} \\
& =(A-f)\left[1+\lambda(2 \rho)^{s}\right]+(g-A)\left[1+p \lambda(2 \rho)^{s}\right] \\
& =(g-f)+\lambda(2 \rho)^{s}(p g-f),
\end{aligned}
$$

donc d'après le côté droit de l'encadrement (1)

$$
\begin{aligned}
\widetilde{\Phi}\left(x, v^{+}\right) \leq \frac{2^{s} \lambda}{M} \rho^{s} & \leq \frac{2^{s} \lambda}{M}(\Delta \varphi+A(p-1) \varphi) \\
& =(\Delta+A(p-1)) v^{+}
\end{aligned}
$$

- De même, soit

$$
v^{-}=-\frac{2^{s}(1-\delta)}{M} \varphi=-(1-\delta)(2 \rho)^{s}
$$

Alors $0>v^{-} \geq-(1-\delta)>-1$ et comme $t \mapsto(1+t)^{p}$ est convexe pour BULLETIN DE LA SOCIÉTÉ MATHÉMATIQUE DE FRANCE 
$p>1$, on a $(1+t)^{p} \leq 1+q t$ pour tout $t \in[-(1-\delta), 0]$; ainsi (en rappelant que $g \leq A)$, on a

$$
\begin{aligned}
\widetilde{\Phi}\left(x, v^{-}\right) \geq & (A-f)\left(1+v^{-}\right)+(g-A)\left(1+q v^{-}\right)+A(p-1) v^{-} \\
= & (A-f)\left[1-(1-\delta)(2 \rho)^{s}\right]+(g-A)\left[1-q(1-\delta)(2 \rho)^{s}\right] \\
& -A(p-1)(1-\delta)\left(2 \rho^{s}\right) \\
=(g-f)+(f-A)(1-\delta)(2 \rho)^{s}+ & q(A-g)(1-\delta)(2 \rho)^{s} \\
& -(1-\delta) A(p-1)(2 \rho)^{s}
\end{aligned}
$$

donc d'après le côté gauche de l'encadrement (1)

$$
\begin{aligned}
\widetilde{\Phi}\left(x, v^{-}\right) \geq-\frac{2^{s}(1-\delta)}{M} \rho^{s} & =-\frac{2^{s}(1-\delta)}{M}(\Delta+A(p-1)) \varphi \\
& =(\Delta+A(p-1)) v^{-} .
\end{aligned}
$$

Pour tout $\beta \in] 0, \alpha[$, il existe donc ( $c f$. [N] par exemple) une solution $v$ de classe $\mathrm{C}_{\text {loc }}^{k+2, \beta}$ de l'équation (E) comprise entre $v^{+}$et $v^{-} ; v$ est donc dans $\Lambda_{0}^{s}$.

En outre, l'opérateur $\Delta+A(p-1)$ est uniformément elliptique sur les compacts de la boule unité; donc, par la théorie de Schauder classique, comme $\widetilde{\Phi}(x, v)$ est dans $\mathrm{C}_{\text {loc }}^{k, \alpha}, v$ est dans $\mathrm{C}_{\text {loc }}^{k+2, \alpha}$.

Remarque. - Comme $v$ est comprise entre $v^{+}$et $v^{-}$, on a l'encadrement :

$$
-1<-(1-\delta) \leq-2^{s}(1-\delta) \rho^{s} \leq v \leq 2^{s} \lambda \rho^{s} \leq \lambda
$$

\subsubsection{Unicité.}

Proposition 2. - Sif et $g$ sont dans $\mathrm{C}_{\mathrm{loc}}^{0}$, avec toujours $A-g \geq 0$, si $v$ est solution de $(\mathrm{E})$ dans $\mathrm{C}_{\mathrm{loc}}^{2} \cap C^{0}(\bar{B}), v>-1$ et $v=0$ sur $\partial B$, alors $v$ est unique.

Preuve. - Soit $u=\ln (1+v)$; alors (E) devient :

$$
\Delta u-|\nabla u|^{2}=A-f+(g-A) \mathrm{e}^{(p-1) u} .
$$

Si $u_{0}$ et $u_{1}$ sont deux telles solutions, soit $w=u_{1}-u_{0}$. Alors :

$$
\Delta w-\left|\nabla u_{1}\right|^{2}+\left|\nabla u_{0}\right|^{2}=(g-A)\left[\mathrm{e}^{(p-1) u_{1}}-\mathrm{e}^{(p-1) u_{0}}\right] .
$$

TOME $125-1997-\mathrm{N}^{\circ} 3$ 
Or nous avons

$$
\nabla^{i} u_{1} \nabla_{i} u_{1}-\nabla^{i} u_{0} \nabla_{i} u_{0}=\nabla^{i}\left(u_{1}+u_{0}\right) \nabla_{i}\left(u_{1}-u_{0}\right)
$$

et

$$
\mathrm{e}^{(p-1) u_{1}}-\mathrm{e}^{(p-1) u_{0}}=(p-1) \int_{0}^{1} \mathrm{e}^{(p-1) u_{t}} \mathrm{~d} t\left(u_{1}-u_{0}\right),
$$

où $u_{t}=t u_{1}+(1-t) u_{0} ;$ ainsi

$$
L w:=\Delta w-\nabla^{i}\left(u_{1}+u_{0}\right) \nabla_{i} w+(A-g)(p-1) \int_{0}^{1} \mathrm{e}^{(p-1) u_{t}} \mathrm{~d} t w=0
$$

et $w=0$ sur $\partial B$. Comme $L$ est elliptique et que son coefficient d'ordre zéro est non-négatif (par hypothèse, $A-g \geq 0$ ), on a nécessairement $w=0$ sur $B$ ( $c f .[\mathrm{GT}$, p. 33 , th. 3.3]).

Remarque. - La solution construite au paragraphe 3.1.1 est donc unique dès que $s>0$.

3.1.3. Régularité. - On suppose $f, g \in \Lambda_{k, \alpha}^{s}$ : que peut-on en déduire sur la solution de l'équation ( $\mathrm{E}$ ) construite précédemment? Pour répondre à cette question, nous avons besoin $\mathrm{du}$ :

LEMme (borne uniforme). - Soient $f, g \in \Lambda_{k, \alpha}^{s}$, avec $s \geq 0$, vérifiant la condition (C) (cf. appendice 6.3, avec $K=A(p-1)$ ) et $0<\alpha<1$. On suppose :

$$
\begin{aligned}
& A>\frac{n-1+s(s-(n-1))}{p-1}, \\
& \inf _{x \in B}\left[(A-g) p\left(1-(1-\delta)(2 \rho)^{s}\right)^{p-1}+f-A-(n-1)\right] \\
& >s(s-(n-1)) .
\end{aligned}
$$

Si $v$ est solution de $(\mathrm{E})$ dans $\Lambda_{k+2, \alpha}^{s}$, avec $-(1-\delta)(2 \rho)^{s} \leq v \leq \lambda(2 \rho)^{s}$, alors $\|v\|_{k+2, \alpha}^{s} \leq C$ où $C$ ne dépend que de $A, f, g, n, s, k, \alpha, \delta, p$.

Preuve. - Dans toute la suite de cet article, pour préciser qu'une constante $C$ est indépendante d'une quantité $v$, nous utiliserons la notation $C(\hat{v})$.

a) On sait ( $c f$. remarque en fin du $\S 3.1 .1$ ) que

$$
\|v\|_{0}^{s} \leq 2^{s} \max ((1-\delta), \lambda)\left\|\rho^{s}\right\|_{0}^{s}=2^{s} \max ((1-\delta), \lambda)=C(\hat{v})
$$


Si on montre que $\|\nabla v\|_{0}^{s-1} \leq C(\hat{v})$, alors $\|v\|_{1}^{s} \leq C(\hat{v})$ et donc, pour tout $\alpha \in] 0,1[$, on aura :

$$
\|v\|_{0, \alpha}^{s} \leq C(\hat{v}) .
$$

Pour montrer cela dérivons l'équation (E) : pour $i=1, . ., n$, nous avons

$$
\begin{aligned}
\nabla_{i} \Delta v=p(1+v)^{p-1}(g-A) \nabla_{i} v+ & (1+v)^{p} \nabla_{i} g \\
& +(A-f) \nabla_{i} v-(1+v) \nabla_{i} f .
\end{aligned}
$$

Or

$$
\nabla_{i} \Delta v=R_{i k} \nabla^{k} v+\Delta \nabla_{i} v=-(n-1) \nabla_{i} v+\Delta \nabla_{i} v
$$

donc

$$
\begin{aligned}
\Delta \nabla_{i} v+\left[(A-g) p(1+v)^{p-1}+f-\right. & A-(n-1)] \nabla_{i} v \\
& =(1+v)^{p} \nabla_{i} g-(1+v) \nabla_{i} f .
\end{aligned}
$$

Notons $K(x)$ le terme d'ordre 0 (terme entre crochets facteur de $\nabla_{i} v$ ); puisque $(A-g) \geq 0$, on a

$$
K(x) \geq(A-g) p\left(1-(1-\delta)(2 \rho)^{s}\right)^{p-1}+f-A-(n-1)
$$

et donc, d'après la condition (3),

$$
\inf _{x \in B} K(x)>s(s-(n-1)) .
$$

Dans ce cas l'estimation «basique» de [GL] est vraie pour $(\Delta+K I)$ agissant sur les 1-tenseurs covariants :

$$
\begin{aligned}
\|\nabla v\|_{0}^{s-1} & \leq C(\widehat{K})\left\|(1+v)^{p} \nabla g-(1+v) \nabla f\right\|_{0}^{s-1} \\
& \leq C(\widehat{K})\left[\left\|(1+v)^{p}\right\|_{0}^{0}\|\nabla g\|_{0}^{s-1}+\|1+v\|_{0}^{0}\|\nabla f\|_{0}^{s-1}\right] \\
& \leq C(\widehat{K})\left[\left(1+\|v\|_{0}^{0}\right)^{p}\|\nabla g\|_{0}^{s-1}+\left(1+\|v\|_{0}^{0}\right)\|\nabla f\|_{0}^{s-1}\right] \\
& \leq C(\widehat{K}) .
\end{aligned}
$$

b) On a donc $\|v\|_{0, \alpha}^{s} \leq C(\hat{v})$. Soit $\ell$ un entier compris entre 0 et $k$, pour lequel on suppose $\|v\|_{\ell, \alpha}^{s} \leq C(\hat{v})$. D'après une estimation de type Schauder sur $B$ qui découle sans difficulté de [GL, p. 206, 209], on a :

$$
\begin{aligned}
\|v\|_{\ell+2, \alpha}^{s} \leq C(\hat{v})\left[\left\|(1+v)^{p}(g-A)+(A-f)(1+v)\right\|_{\ell, \alpha}^{s}+\|v\|_{0}^{s}\right] \\
\leq C(\hat{v})\left[\|\left((1+v)^{p}-1\right)(g-A)\right. \\
\left.+(A-f) v+(g-f)\left\|_{\ell, \alpha}^{s}+\right\| v \|_{0}^{s}\right] .
\end{aligned}
$$

TOME $125-1997-\mathrm{N}^{\circ} 3$ 
En utilisant le lemme de l'appendice 6.1 (une estimation non linéaire) avec la fonction

$$
\Psi(t)=(1+t)^{p}-1, \quad a=1-\delta \quad \text { et } \quad b=\lambda
$$

(c'est ici qu'intervient la condition $s \geq 0$ ), on obtient :

$$
(1+v)^{p}-1 \in \Lambda_{\ell, \alpha}^{s}, \quad\left\|(1+v)^{p}-1\right\|_{\ell, \alpha}^{s} \leq C(\hat{v}) .
$$

Ainsi, compte tenu de [GL, prop. 3.3, p. 208],

$$
\begin{aligned}
\|v\|_{\ell+2, \alpha}^{s} \leq C(\hat{v})\left[\left\|(1+v)^{p}-1\right\|_{\ell, \alpha}^{s}\|g-A\|_{\ell, \alpha}^{0}\right. & \\
& \left.+\|v\|_{\ell, \alpha}^{s}\|A-f\|_{\ell, \alpha}^{0}+\|g-f\|_{\ell, \alpha}^{s}+\|v\|_{0}^{s}\right],
\end{aligned}
$$

soit finalement $\|v\|_{\ell+2, \alpha}^{s} \leq C(\hat{v})$.

Ainsi par récurrence (nous sommes partis de $\ell=0$ ) :

$$
\|v\|_{k+2, \alpha}^{s} \leq C(\hat{v}) .
$$

Nous allons maintenant pouvoir répondre à la question posée au début de cette section en redémontrant en fait directement (par la méthode de continuité) l'existence d'une unique solution avec la bonne «régularité pondérée».

Proposition 3.-On suppose $f, g \in \Lambda_{k, \alpha}^{s}, s>0$ vérifiant (C), toujours $g \leq A$, la condition (2) $A>(n-1+s(s-(n-1))) /(p-1)$ et

$$
\begin{aligned}
\inf _{x \in B}[(A-|g|) p(1- & \left.\left.(1-\delta)(2 \rho)^{s}\right)^{p-1}-|f|-A-(n-1)\right] \\
& >s(s-(n-1)), \\
-(1-\delta)\left[\frac{1}{M}-A(p-1)+q(A-|g|)-(A+|f|)\right] & \\
\leq & (2 \rho)^{-s}|g-f| \leq \lambda\left(\frac{1}{M}-p|g|-|f|\right),
\end{aligned}
$$

avec $M=M(n, s, A(p-1))$ (cf. appendice 6.3 avec $K=A(p-1))$.

Alors l'unique solution $v \in \Lambda_{0}^{s} \cap C_{\mathrm{loc}}^{k+2, \alpha}$ de l'équation (E) avec $v>-1$ est en fait dans $\Lambda_{k+2, \alpha}^{s}$. De plus, $v$ tend vers 0 dans $\Lambda_{k+2, \alpha}^{s}$ quand $f, g$ tendent vers 0 dans $\Lambda_{k, \alpha}^{s}$.

Remarque. - On a les implications (4) $\Rightarrow(3)$ et $(5) \Rightarrow(1)$. 
Preuve. - Considérons pour $t \in[0,1]$ la famille d'équations :

$$
\Delta v=(t g-A)(1+v)^{p}+(A-t f)(1+v), \quad v>-1
$$

et soit

$$
\mathcal{S}=\left\{t \in[0,1] ; \exists v_{t} \in \Lambda_{k+2, \alpha}^{s} \text { solution de }\left(\mathrm{E}_{t}\right) \text { avec } v_{t}>-1\right\} .
$$

Nous allons montrer que $\mathcal{S}=[0,1]$ par un argument de connexité; dès lors, la partie «existence» de la proposition sera démontrée pour l'équation $(\mathrm{E})$ qui n'est autre que $\left(\mathrm{E}_{1}\right)$. L'unicité a lieu en vertu de notre proposition 2 du paragraphe 3.1.2.

La continuité par rapport à $f$ et $g$ au voisinage de zéro découle de l'argument d'inversion locale utilisé ci-après (en l'appliquant à $t_{0}=0$ ).

a) L'ensemble $\mathcal{S}$ n'est pas vide car 0 appartient à $\mathcal{S}$ (en effet, on peut prendre $v_{0}=0$ ).

b) L'ensemble $\mathcal{S}$ est ouvert dans [0,1]. Soit $\Psi:[0,1] \times \Lambda_{k+2, \alpha}^{s} \rightarrow \Lambda_{k, \alpha}^{s}$ définie par :

$$
\Psi(t, v)=\Delta v-(t g-A)(1+v)^{p}-(A-t f)(1+v) .
$$

Si on montre que $\partial \Psi / \partial v\left(t_{0}, v_{0}\right)$ est un isomorphisme de $\Lambda_{k+2, \alpha}^{s}$ sur $\Lambda_{k, \alpha}^{s}$ pour tout couple $\left(t_{0}, v_{0}\right) \in[0,1] \times \Lambda_{k+2, \alpha}^{s}$ solution de $\Psi\left(t_{0}, v_{0}\right)=0$ avec $v_{0}>-1$, alors il suit du théorème des fonctions implicites l'existence d'un $\epsilon>0$ tel que pour tout $t \in] t_{0}-\epsilon, t_{0}+\epsilon\left[\cap[0,1]\right.$, il existe $v_{t} \in \Lambda_{k+2, \alpha}^{s}$ solution de $\Psi(t, v)=0$ avec $v>-1$; dans ce cas on aura bien $\mathcal{S}$ ouvert relativement à $[0,1]$. Calculons :

$$
\frac{\partial \Psi}{\partial v}\left(t_{0}, v_{0}\right) \delta v=\Delta \delta v-\left(t_{0} g-A\right) p\left(1+v_{0}\right)^{p-1} \delta v+\left(t_{0} f-A\right) \delta v .
$$

Les conditions (4) et (5) étant vérifiées pour $f$ et $g$ et tout $t \in[0,1]$, elles le sont encore pour $t f$ et $t g$; donc (3) et (1) aussi. Ainsi par unicité (prop. 2 $\mathrm{du} \S 3.1 .2)$ et d'après la remarque qui suit la prop. $1 \mathrm{du} \S 3.1 .1$, pour tout couple $\left(t, v_{t}\right) \in[0,1] \times \Lambda_{k+2, \alpha}^{s}$ solution de $\Psi\left(t, v_{t}\right)=0$ avec $v_{t}>-1$, on a l'estimation uniforme $v_{t} \geq-2^{s}(1-\delta) \rho^{s}$; celle-ci est donc vérifiée par $v_{0}$. Par ailleurs :

$$
\begin{aligned}
K_{0} & :=-\left(t_{0} g-A\right) p\left(1+v_{0}\right)^{p-1}+\left(t_{0} f-A\right) \\
& \geq\left(A-t_{0}|g|\right) p\left[1-(1-\delta)(2 \rho)^{s}\right]^{p-1}-A-t_{0}|f| \\
& \geq(A-|g|) p\left[1-(1-\delta)(2 \rho)^{s}\right]^{p-1}-A-|f| \\
& \geq \inf _{x \in B}\left[(A-|g|) p\left(1-(1-\delta)(2 \rho)^{s}\right)^{p-1}-A-|f|\right] \\
& >s(s-(n-1)), \text { d'après notre condition (4). }
\end{aligned}
$$

TOME $125-1997-\mathrm{N}^{\circ} 3$ 
Le théorème 3.10 de [GL, p. 217] affirme alors que $\Delta+K_{0}$ est bien un isomorphisme de $\Lambda_{k+2, \alpha}^{s}$ sur $\Lambda_{k, \alpha}^{s}$.

c) L'ensemble $\mathcal{S}$ est fermé. Soit $\left\{t_{i}\right\}_{i \in \mathbb{N}}$ une suite de $\mathcal{S}$ qui tend vers $t \in[0,1]$; on veut montrer que $t$ appartient à $\mathcal{S}$. Notons $v_{i}=v_{t_{i}}$; par le lemme $1 \mathrm{du} \oint 3.1 .3$, les $v_{i}$ sont uniformément bornés dans $\Lambda_{k+2, \alpha}^{s}$ (en effet, l'équation $\left(\mathrm{E}_{t}\right)$ n'est autre que l'équation $(\mathrm{E})$ pour $t f$ et $t g$, comme la constante $C$ ne fait intervenir que des sommes et des produits de $\|f\|$ et $\|g\|$, et que $\|t h\| \leq\|h\|$ pour $t \in[0,1]$, les $v_{i}$ auront la même borne $C$ ).

Ainsi, d'après le lemme de l'injection compacte (voir appendice 6.2 ci-après), il existe pour tout $t \in] 0, s$ [ et tout $\beta \in] 0, \alpha[$ une soussuite renotée $v_{i}$ qui converge dans $\Lambda_{k+2, \beta}^{t}$ vers une fonction $v \in \Lambda_{k+2, \alpha}^{s}$ avec $v \geq-1$.

De plus, pour tout $i, \Psi\left(t_{i}, v_{i}\right)=0$ et $\Psi$ est encore continue sur $[0,1] \times \Lambda_{k+2, \beta}^{t} ;$ donc $0=\lim _{i \rightarrow \infty} \Psi\left(t_{i}, v_{i}\right)=\Psi(t, v)$ : ainsi $v$ vérifie $\left(E_{t}\right)$. Notons pour conclure la minoration uniforme $v_{i} \geq-(1-\delta)$, qui a lieu par unicité et s'applique encore à $v$; donc $v>-1$ et $t \in \mathcal{S}$.

\subsection{Application géométrique (dimension $n \geq 3$ ).}

Soit $H$ une métrique conforme à la métrique hyperbolique $H_{0}$, écrite sous la forme $H=(1+v)^{\frac{4}{n-2}} H_{0}$. Sa courbure scalaire s'écrit (voir par exemple [A, p. 126]) :

$$
\operatorname{Scal}(H)=(1+v)^{-\frac{n+2}{n-2}}\left[4 \frac{n-1}{n-2} \Delta v+S_{0}(1+v)\right],
$$

où $S_{0}=\operatorname{Scal}\left(H_{0}\right)$, et prescrire $\operatorname{Scal}(H)=S_{0}+\sigma$ équivaut à chercher $H$ sous la forme précédente avec $v$ solution sur $B$ de l'équation (E) :

$$
\Delta v=(A-f)(1+v)+(g-A)(1+v)^{p},
$$

avec :

$$
\begin{aligned}
A & =-\frac{n-2}{4(n-1)} S_{0}=\frac{n(n-2)}{4} \quad \text { car } S_{0}=-n(n-1), \\
p & =\frac{n+2}{n-2}, \quad \text { donc ici } A(p-1)=n, \\
g & =\frac{n-2}{4(n-1)} \sigma, \\
f & =0 .
\end{aligned}
$$

Notons qu'on a $p>1$. Par ailleurs, $s$ vérifie la condition (C) de l'appendice 6.3 avec $K=A(p-1)=n$ si et seulement si $s$ est dans ] $-1, n[$. 
Enfin :

$$
A=\frac{n}{p-1}>\frac{n-1+s(s-(n-1))}{p-1}
$$

dès que $s$ appartient à $] s_{-}, s_{+}\left[\right.$où $s_{ \pm}=\frac{1}{2}\left(n-1 \pm \sqrt{(n-1)^{2}+4}\right)$. Notons que $s_{-}$est dans ] $-1,0\left[\right.$ et que $s_{+}$est dans $] n-1, n[$.

L'étude précédente de l'équation (E) jointe au corollaire de l'appendice 6.3 conduit au :

ThÉorème 3. - S'il existe $\delta \in] 0,1[$ et $\lambda \in \mathbb{R}, \lambda \geq 0$ tels que, en notant $q=\frac{1-\delta^{p}}{1-\delta}$, la fonction $\sigma \in \Lambda_{0}^{s} \cap C_{\mathrm{loc}}^{k, \alpha}($ avec $0 \leq s<n$ et $0<\alpha<1)$ vérifie $S_{0}+\sigma \leq 0$ et l'encadrement:

$$
\begin{aligned}
-(1-\delta)\left[\frac{1}{M}-n+\right. & \left.q\left(\frac{n(n-2)}{4}-\frac{n-2}{4(n-1)} \sigma\right)-\frac{n(n-2)}{4}\right] \\
\leq & (2 \rho)^{-s} \frac{n-2}{4(n-1)} \sigma \leq \lambda\left(\frac{1}{M}-\frac{n+2}{4(n-1)} \sigma\right)
\end{aligned}
$$

où $M=M(n, s, n)$ (cf. appendice 6.3), alors il existe $v \in \Lambda_{0}^{s} \cap C_{\mathrm{loc}}^{k+2, \alpha}$ telle que la métrique conforme $H=(1+v)^{\frac{4}{n-2}} H_{0}$ admette $S_{0}+\sigma$ pour courbure scalaire et $v$ est unique si $s>0$.

Si de plus $\sigma \in \Lambda_{k, \alpha}^{s}$ avec $\left.s \in\right] 0, s_{+}[$vérifie

$$
\begin{aligned}
-(1-\delta)\left[\frac{1}{M}-n+\right. & \left.q\left(\frac{n(n-2)}{4}-\frac{n-2}{4(n-1)}|\sigma|\right)-\frac{n(n-2)}{4}\right] \\
\leq & (2 \rho)^{-s} \frac{n-2}{4(n-1)}|\sigma| \leq \lambda\left(\frac{1}{M}-\frac{n+2}{4(n-1)}|\sigma|\right)
\end{aligned}
$$

et

$$
\begin{aligned}
\inf _{x \in B}\left[\left(\frac{n(n-2)}{4}-\frac{n-2}{4(n-1)} \mid\right.\right. & \sigma \mid) \frac{n+2}{n-2}\left(1-(1-\delta)(2 \rho)^{s}\right)^{\frac{4}{n-2}} \\
& \left.-\frac{n(n-2)}{4}-(n-1)\right]>s(s-(n-1)),
\end{aligned}
$$

alors $v$ appartient $\grave{a} \Lambda_{k+2, \alpha}^{s}$.

Remarque. - L'obtention de ce résultat global nous a coûté une diminution de l'intervalle $-1<s<n$ nécessaire pour le résultat local du paragraphe 2 (théorème 1 ). 


\section{Courbure scalaire conforme en dimension deux}

Toujours sur $B$ munie de la métrique hyperbolique $H_{0}=\rho^{-2} E$, on considère à présent l'équation :

$$
\Delta v=\mathrm{e}^{v}(f-A)+A,
$$

où $A \in \mathbb{R}^{+}$et $f$ est une fonction donnée sur $B$.

Nous allons montrer l'existence d'une solution, son unicité puis sa régularité, ceci en toute dimension; pour cela nous procédons comme au paragraphe 3. Ensuite nous appliquerons ce résultat au cas particulier de la prescription de la courbure scalaire conforme en dimension 2 .

Remarque. - Dans la version «thèse» de ce travail, nous obtenons pour ce chapitre 4 des résultats où le paramètre de pondération $s$ peut évoluer dans des intervalles un peu plus larges moyennant des inégalités plus contraignantes sur $f, A$ et $\sigma$ (qu'il nous a paru trop lourd de reproduire ici), en utilisant pour cela une réécriture de $\left(E^{\prime}\right)$ sous la forme

$$
(\Delta+A) v=\mathrm{e}^{v}(f-A)+A(1+v) .
$$

\section{1 Étude EDP (en dimension n quelconque).}

4.1.1. Existence. - Soit $M=M(n, s, 0)$ la constante de l'appendice 6.3 ci-après (dans lequel nous démontrons que la fonction positive $\varphi=M \rho^{s}$ vérifie sur tout $B$ l'inéquation $\Delta \varphi \geq \rho^{s}$ ).

Proposition 4. - Soient $\left.f \in \Lambda_{0}^{s} \cap C_{\mathrm{loc}}^{k, \alpha}, s \in\right] 0, n-1[$ et $\alpha \in] 0,1\left[\right.$. $S^{\prime} i l$ existe $\lambda \geq 0$ tel que

$$
f \leq \frac{\lambda \rho^{s}-A}{\mathrm{e}^{\lambda \varphi}}+A
$$

alors il existe une solution $v$ de $\left(\mathrm{E}^{\prime}\right)$ dans $\Lambda_{0}^{s} \cap C_{\mathrm{loc}}^{k+2, \alpha}$. En outre :

$$
-2^{s} \varphi\|f\|_{0}^{s} \leq v \leq \lambda \varphi
$$

Preuve. - Cherchons une sur-solution et une sous-solution de l'équation $\left(\mathrm{E}^{\prime}\right)$. Soit $v^{+}=\lambda \varphi$; on a :

$$
\Delta v^{+} \geq \lambda \rho^{s} \geq(f-A) \mathrm{e}^{\lambda \varphi}+A,
$$

la deuxième inégalité étant vérifiée d'après l'hypothèse de la proposition.

Soit $v^{-}=-2^{s}\|f\|_{0}^{s} \varphi$. Déjà, comme $\varphi \geq 0, v^{-}$est non-positive; donc on a bien $v^{-} \leq v^{+}$. Et comme

$$
f \geq-2^{s}\|f\|_{0}^{s} \rho^{s}=-A-2^{s}\|f\|_{0}^{s} \rho^{s}+A \geq \frac{-A-2^{s}\|f\|_{0}^{s} \rho^{s}}{\mathrm{e}^{-2^{s}\|f\|_{0}^{s} \rho^{s}}}+A
$$


on a aussi :

$$
\Delta v^{-} \leq-2^{s}\|f\|_{0}^{s} \rho^{s} \leq \mathrm{e}^{-2^{s}\|f\|_{0}^{s} \rho^{s}}(f-A)+A=\mathrm{e}^{v^{-}}(f-A)+A .
$$

Ainsi (voir e.g. $[\mathrm{N}]$ ), il existe $v \in C_{\mathrm{loc}}^{k+2, \alpha}$ comprise entre $v^{+}$et $v^{-}$, donc dans $\Lambda_{0}^{s}$, solution de l'équation $\left(\mathrm{E}^{\prime}\right)$.

\subsubsection{Unicité.}

Proposition 5. - Si $f \in C_{\mathrm{loc}}^{0}$ et $f \leq A$, alors l'équation ( $\left.\mathrm{E}^{\prime}\right)$ admet au plus une solution $v$ dans $C_{\mathrm{loc}}^{2} \cap C^{0}(\bar{B})$ nulle sur $\partial B$.

Preuve. - Soient $v_{0}$ et $v_{1}$ deux telles solutions et $w=v_{1}-v_{0}$, alors :

$$
\Delta w=\left(\mathrm{e}^{v_{1}}-\mathrm{e}^{v_{0}}\right)(f-A)=(f-A)\left[\int_{0}^{1} \mathrm{e}^{t v_{1}+(1-t) v_{0}} \mathrm{~d} t\right]\left(v_{1}-v_{0}\right)
$$

Donc $w$ vérifie

$$
\Delta w+(f-A)\left[\int_{0}^{1} \mathrm{e}^{t v_{1}+(1-t) v_{0}} \mathrm{~d} t\right] w=0
$$

dans $B$, avec $w=0$ sur $\partial B$. Le coefficient de $w$ est non-négatif puisque $(A-f) \geq 0$; le principe du maximum (voir [GT, p. 33, th. 3.3]) implique $w=0$.

Remarque. - Si $f \leq A$, on peut prendre $\lambda=2^{s}\|f\|_{0}^{s}$ dans la proposition $5 \mathrm{du} \S 4.1 .1$. En effet prenons $v^{+}=2^{s}\|f\|_{0}^{s} \varphi$ alors

$$
\Delta v^{+} \geq 2^{s}\|f\|_{0}^{s} \rho^{s} \geq f \geq f+\left(\mathrm{e}^{v^{+}}-1\right)(f-A)=\mathrm{e}^{v^{+}}(f-A)+A .
$$

Dans ce cas, la solution de $\left(\mathrm{E}^{\prime}\right)$ vérifie l'estimation

$$
|v| \leq 2^{s} \varphi\|f\|_{0}^{s} .
$$

\subsubsection{Régularité.}

Proposition 6. - En supposant $\left.f \in \Lambda_{k, \alpha}^{s}, s \in\right] 0, n-1[, \alpha \in] 0,1[$, $A \geq(n-1)+s(s-(n-1)), f \leq A$, et

$$
\inf _{x \in B}\left[\mathrm{e}^{-2^{s}\|f\|_{0}^{s} \varphi}(A-|f|)-(n-1)\right]>s(s-(n-1)),
$$

alors la solution $v$ de l'équation $\left(\mathrm{E}^{\prime}\right)$ est dans $\Lambda_{k+2, \alpha}^{s}$. De plus, $v$ tend vers 0 dans $\Lambda_{k+2, \alpha}^{s}$ quand $f$ tend vers 0 dans $\Lambda_{k, \alpha}^{s}$.

Remarque. - Si $n \leq 5$, alors la condition (6) implique $|f| \leq A$.

$$
\text { TOME } 125-1997-\mathrm{N}^{\circ} 3
$$


Preuve. - D'après les propositions 4 et 5 précédentes et l'avant-dernière remarque, il existe bien une solution unique de $\left(\mathrm{E}^{\prime}\right)$ dans $\Lambda_{0}^{s} \cap C_{\mathrm{loc}}^{k+2, \alpha}$. On va montrer qu'elle est dans $\Lambda_{k+2, \alpha}^{s}$ par la méthode de continuité; l'argument d'inversion locale utilisé ci-après montre (appliqué en $t_{0}=0$ ) que $v$ tend vers 0 quand $f$ tend vers 0 .

Soit $\Psi:[0,1] \times \Lambda_{k+2, \alpha}^{s} \rightarrow \Lambda_{k, \alpha}^{s}$ définie par :

$$
\Psi(t, v)=\Delta v-\mathrm{e}^{v}(t f-A)-A,
$$

et soit

$$
\mathcal{S}=\left\{t \in[0,1] ; \exists v_{t} \in \Lambda_{k+2, \alpha}^{s} \text { solution de } \Psi(t, v)=0\right\}
$$

a) L'ensemble $\mathcal{S}$ n'est pas vide car 0 appartient à $\mathcal{S}$ (en effet, on a $\Psi(0,0)=0)$.

b) L'ensemble $\mathcal{S}$ est ouvert dans $[0,1]$. Soient $t_{0} \in \mathcal{S}$ et $v_{0}=v_{t_{0}}$ appartenant à $\Lambda_{k+2, \alpha}^{s}$; alors :

$$
\frac{\partial \Psi}{\partial v}\left(t_{0}, v_{0}\right) \delta v=\Delta \delta v+\left(A-t_{0} f\right) \mathrm{e}^{v_{0}} \delta v
$$

Quand $f \geq 0$, on a $t_{0} f \leq f \leq A$ et quand $f \leq 0$, on a $t_{0} f \leq 0<A$; donc on a toujours $\left(A-t_{0} f\right) \mathrm{e}^{v_{0}} \geq 0$ et d'après [GL, p. 217]

$$
\Delta+\left(A-t_{0} f\right) \mathrm{e}^{v_{0}}
$$

est un isomorphisme de $\Lambda_{k+2, \alpha}^{s}$ dans $\Lambda_{k, \alpha}^{s}$ sous l'hypothèse $0>s(s-(n-1))$ vérifiée pour $s \in] 0, n-1[$. D'après le théorème des fonctions implicites, il existe donc $\epsilon>0$ tel que pour tout $t \in] t_{0}-\epsilon, t_{0}+\epsilon[\cap[0,1]$, il existe $v_{t} \in \Lambda_{k+2, \alpha}^{s}$ solution de $\Psi\left(t, v_{t}\right)=0$. Par suite $\mathcal{S}$ est ouvert dans $[0,1]$.

c) L'ensemble $\mathcal{S}$ est fermé. Comme précédemment ( $c f$. fin du $\S 3.1 .3$ ), il suffit de majorer uniformément les solutions de $\left(\mathrm{E}^{\prime}\right)$.

Pour cela, dérivons l'équation $\left(\mathrm{E}^{\prime}\right): \nabla_{i} \Delta v=\mathrm{e}^{v}(f-A) \nabla_{i} v+\mathrm{e}^{v} \nabla_{i} f$ où $\nabla_{i} \Delta v=-(n-1) \nabla_{i} v+\Delta \nabla_{i} v$; donc :

$$
\Delta \nabla_{i} v+\left[\mathrm{e}^{v}(A-f)-(n-1)\right] \nabla_{i} v=\mathrm{e}^{v} \nabla_{i} f
$$

D'après (6) et la remarque qui suit l'unicité $(\S 4.1 .2)$, on a :

$$
\begin{aligned}
\mathrm{e}^{v}(A-f) & -(n-1) \geq \mathrm{e}^{-2^{s}\|f\|_{0}^{s} \varphi}(A-|f|)-(n-1) \\
& \geq \inf _{x \in B}\left[\mathrm{e}^{-2^{s}\|f\|_{0}^{s} \varphi}(A-|f|)-(n-1)\right]>s(s-(n-1)) .
\end{aligned}
$$


Donc l'estimation de base de [GL] a lieu :

$$
\begin{aligned}
\|\nabla v\|_{0}^{s-1} & \leq C(\hat{v})\left\|\mathrm{e}^{v} \nabla f\right\|_{0}^{s-1} \\
& \leq C(\hat{v})\left\|\mathrm{e}^{v}\right\|_{0}^{0}\|\nabla f\|_{0}^{s-1} \\
& \leq C(\hat{v}) \mathrm{e}^{\|v\|_{0}^{0}}\|\nabla f\|_{0}^{s-1}=C(\hat{v}) .
\end{aligned}
$$

Ainsi $v$ est dans $\Lambda_{0, \alpha}^{s}$ et $\|v\|_{0, \alpha}^{s} \leq C(\hat{v})$. D'après l'estimation de type Schauder qui découle de [GL, p. 206, 209], si $v \in \Lambda_{\ell, \alpha}^{s}$ avec $0 \leq \ell \leq k$,

$$
\|v\|_{\ell+2, \alpha}^{s} \leq C(\hat{v})\left[\left\|\left(\mathrm{e}^{v}-1\right)(f-A)+f\right\|_{\ell, \alpha}^{s}+\|v\|_{0}^{s}\right] .
$$

En utilisant l'appendice 6.1 avec $\Psi(t)=\mathrm{e}^{t}-1$ et $a=b=2^{s} M\|f\|_{0}^{s}$, on a :

$$
\mathrm{e}^{v}-1 \in \Lambda_{\ell, \alpha}^{s}, \quad\left\|\mathrm{e}^{v}-1\right\|_{\ell, \alpha}^{s} \leq C(\hat{v}) .
$$

Ainsi

$$
\|v\|_{\ell+2, \alpha}^{s} \leq C(\hat{v})\left[\left\|\left(\mathrm{e}^{v}-1\right)\right\|_{\ell, \alpha}^{s}\|A-f\|_{\ell, \alpha}^{0}+\|f\|_{\ell, \alpha}^{s}+\|v\|_{0}^{s}\right] \leq C(\hat{v}) .
$$

On conclut alors comme précédemment ( $\S 3.1 .3)$.

\subsection{Application géométrique (dimension $n=2$ ).}

En dimension $n=2$, si $H=\mathrm{e}^{2 g} H_{0}$, alors

$$
\operatorname{Scal}(H)=\mathrm{e}^{-2 g}\left(S_{0}+2 \Delta g\right)
$$

où $S_{0}=\operatorname{Scal}\left(H_{0}\right)=-2 ;$ donc si l'on prescrit $\operatorname{Scal}(H)=S_{0}+\sigma$, chercher $g$ revient à résoudre :

$$
2 \Delta g=\mathrm{e}^{2 g}\left(S_{0}+\sigma\right)-S_{0} .
$$

On est donc conduit à regarder $\left(\mathrm{E}^{\prime}\right)$ avec

$$
A=-S_{0}=2, \quad v=2 g, \quad f=\sigma .
$$

Ici en outre $s$ est dans $] 0,1\left[\right.$ et $\varphi=\frac{1}{s(s-1)} \rho^{s}$. L'étude précédente nous
donne le :

ThÉorème 4. - Si $\sigma$ est dans $\Lambda_{0}^{s} \cap C_{\mathrm{loc}}^{k, \alpha}$, avec $0<s<1,0<\alpha<1$, et s'il existe $\lambda \geq 0$ tel que

$$
\sigma \leq \frac{\lambda \rho^{s}-2}{\mathrm{e}^{\lambda \varphi}}+2
$$

alors il existe une métrique conforme $H=\mathrm{e}^{2 g} H_{0}$ admettant $S=S_{0}+\sigma$ TOME $125-1997-\mathrm{N}^{\circ} 3$ 
pour courbure scalaire, et asymptotique à $H_{0}$ avec un facteur conforme $2 g$ appartenant à $\Lambda_{0}^{s} \cap C_{\mathrm{loc}}^{k+2, \alpha}$, et vérifiant $-2^{s} \varphi\|\sigma\|_{0}^{s} \leq 2 g \leq \lambda \varphi$.

Si de plus $S \leq 0$, alors $H=\mathrm{e}^{2 g} H_{0}$ est unique et $|2 g| \leq 2^{s} \varphi\|\sigma\|_{0}^{s}$. Enfin, si $\sigma=S-S_{0} \in \Lambda_{k, \alpha}^{s}$, avec $0<s<1,0<\alpha<1$, et si

$$
\inf _{x \in B}\left[\mathrm{e}^{-2^{s} \varphi\|\sigma\|_{0}^{s}}(2-|\sigma|)-1\right]>s(s-1)
$$

alors $g$ appartient $\grave{a} \Lambda_{k+2, \alpha}^{s}$.

\section{Un exemple de sortie de l'intervalle des poids isomorphiques}

Dans ce paragraphe, nous considérons l'équation de la courbure scalaire conforme dans le cas où l'exposant du poids est un réel $s>n+1$.

Proposition 7. - On suppose $n \geq 6, s>n+1$ et qu'il existe un $\epsilon>0$ tel que $\sigma \geq \epsilon \rho^{s}$. Alors, si $v$ est solution de l'équation

$$
\operatorname{Scal}\left[(1+v)^{\frac{4}{n-2}} H_{0}\right]=S_{0}+\sigma
$$

avec $v=0$ sur $\partial B$ et $-1<v \leq 1$, $v$ ne peut appartenir $\grave{a} \Lambda_{0}^{k}$ si $k>n+1$.

Preuve. - Rechercher une métrique $H$ sous la forme $H=(1+v)^{\frac{4}{n-2}} H_{0}$ ayant pour courbure scalaire $S_{0}+\sigma=-n(n-1)+\sigma$ revient à résoudre l'équation :

$$
4 \frac{n-1}{n-2} \Delta v=n(n-1)(1+v)+(\sigma-n(n-1))(1+v)^{p}
$$

en notant $p=(n+2) /(n-2)$ qui est dans l'intervalle [1,2] car $n \geq 6$. Remarquons que $v \geq 0 ; \operatorname{sinon} 0>\inf _{B} v=v\left(x_{0}\right)$ implique $\Delta v\left(x_{0}\right) \leq 0$, ce qui, joint à $\sigma \geq 0$, permet de déduire de l'équation en $x_{0}$ l'implication $1 \leq(1+v)^{p-1} \Rightarrow v\left(x_{0}\right) \geq 0$ qui est une contradiction.

L'équation peut s'écrire :

$$
\Delta v+\left[n+\frac{n}{2}\left(\frac{n+2}{n-2}\right) v\right] v=\frac{n-2}{4(n-1)} \sigma(1+v)^{p}+G(v)
$$

où l'examen de la fonction

$$
v \geq 0 \longmapsto G(v)=-\frac{1}{4} n(n-2)\left[(1+v)^{p}-1-p v-\frac{1}{2} p(p-1) v^{2}\right]
$$

permet de s'assurer que $G(v) \geq 0$ si $p$ est dans $[1,2]$ (et $v \geq 0$ ). 
D'autre part, comme $n \geq 4$ et $v \leq 1$, on a :

$$
2 n+2 \geq n+\frac{n}{2} \frac{n+2}{n-2} \geq n+\frac{n}{2} \frac{n+2}{n-2} v .
$$

Comme $v \geq 0$, on a finalement :

$$
\begin{aligned}
(\Delta+2 n+2) v & \geq \Delta v+\left(n+\frac{n}{2} \frac{n+2}{n-2} v\right) v \\
& =\frac{n-2}{4(n-1)} \sigma(1+v)^{p}+G(v) \\
& \geq \frac{n-2}{4(n-1)} \sigma \geq \frac{n-2}{4(n-1)} \epsilon \rho^{s} .
\end{aligned}
$$

Ainsi par les corollaires 3 et 4 ci-après et le principe du maximum, on a $v \geq \frac{n-2}{4(n-1)} \epsilon \Phi(\rho)$ et donc $v$ est au mieux dans $\Lambda_{0}^{n+1}$.

REMARQUE. - Si on suppose en outre $\sigma \in \Lambda_{0}^{s} \cap C_{\text {loc }}^{k, \alpha}$ dans la proposition 7 , nos résultats d'existence locale (l'inversion locale, th. 1), et globale (th. 3, première partie) fournissent une solution $v \in \Lambda_{0}^{t} \cap C_{\text {loc }}^{k+2, \alpha}$ pour tout $t \in]-1, n$ [, qui vérifie bien $-1<v \leq 1$ dès que $\sigma$ est prise assez petite (en particulier, $\sigma$ telle que $\lambda \leq 1$, cf. th. 3). La proposition précédente affirme alors que cette solution $v$ ne peut être dans $\Lambda_{0}^{s}$ : il y a perte de poids.

Pour établir les corollaires que nous venons d'utiliser, commençons par démontrer un lemme de perte de poids :

Lemme 2. - Soit un réel $K \geq-\frac{1}{4}(n-1)^{2}$ tel que

$$
s_{2}=\frac{1}{2}\left(n-1+\sqrt{(n-1)^{2}+4 K}\right)>0 ;
$$

si $s=s_{2}+p$ avec $p \in \mathbb{N}$ et $p \neq 0$, alors la solution de $(\Delta+K) \varphi=\rho^{s}$ qui s'annule sur $\partial B$ est dans $\Lambda_{0}^{k}$ si et seulement si $k \leq s_{2}$.

Preuve. - On cherche la solution sous la forme $\varphi(x)=\Phi(\rho(x))$ (radiale) avec, pour l'instant formellement :

$$
\Phi^{\prime}(\rho)=\rho^{s_{2}-1} \sum_{j \geq 0} b_{j} \rho^{j}, \quad \Phi(\rho)=\rho^{s_{2}} \sum_{j \geq 0} \frac{b_{j}}{j+s_{2}} \rho^{j} .
$$

TOME $125-1997-\mathrm{N}^{\circ} 3$ 
Alors, d'après le lemme 8 de l'appendice 6.3 :

$$
\begin{aligned}
& (\Delta+K) \Phi(\rho) \\
& =\rho^{s_{2}}\left[\sum_{j \geq 1} 2 b_{j-1}\left(j+s_{2}-2\right) \rho^{j}-\sum_{j \geq 0} b_{j}\left(j+s_{2}-1\right) \rho^{j}\right. \\
& \left.\quad+(4-n) \sum_{j \geq 1} b_{j-1} \rho^{j}+(n-2) \sum_{j \geq 0} b_{j} \rho^{j}+K \sum_{j \geq 0} \frac{b_{j}}{j+s_{2}} \rho^{j}\right] \\
& \quad b_{0}\left(n-s_{2}-1+\frac{K}{s_{2}}\right) \rho^{s_{2}} \\
& \quad+\rho^{s_{2}} \sum_{j \geq 1}\left[\left(2 j+2 s_{2}-n\right) b_{j-1}+\left(-j-s_{2}+n-1+\frac{K}{j+s_{2}}\right) b_{j}\right] \rho^{j} \\
& \quad \rho^{s_{2}} \sum_{j \geq 1}\left[\left(2 j+2 s_{2}-n\right) b_{j-1}+\frac{j\left(-j-2 s_{2}+n-1\right)}{j+s_{2}} b_{j}\right] \rho^{j}
\end{aligned}
$$

car $s_{2}$ est racine de $s(s-(n-1))-K=0$.

Donc $(\Delta+K) \Phi(\rho)=\rho^{s}=\rho^{s_{2}} \rho^{p}$ si et seulement si :

$$
\begin{cases}b_{j}=0 & \text { pour } j \geq p \\ b_{p-1}=\frac{1}{2 p+2 s_{2}-n}=\frac{1}{2 s-n} & \text { pour } j=p-1, \\ b_{j-1}=\frac{j\left(j+2 s_{2}-n+1\right)}{\left(j+s_{2}\right)\left(2 j+2 s_{2}-n\right)} b_{j} & \text { pour } j \in\{1, . ., p-1\} .\end{cases}
$$

\section{Remarques.}

Comme $s_{2} \geq \frac{1}{2}(n-1)$ et $p \geq 1$, alors $b_{j}>0$ pour tout $j<p$. On a toujours $s_{2}>0$ lorsque $n \neq 1$ ou $K \neq 0$.

On obtient ainsi une solution polynomiale en $\rho$ :

$$
\Phi(\rho)=\rho^{s_{2}} \sum_{j=0}^{p-1} \frac{b_{j}}{j+s_{2}} \rho^{j}
$$

qui s'annule sur $\partial B$. Une telle solution est unique par le principe du maximum (cf. [GT, p. 33, th. 3.3]). Elle est dans $\Lambda_{0}^{s_{2}}$ et ne peut donc pas «s'écraser» plus vite à l'infini.

De ce lemme suivent immédiatement les corollaires suivants. 
Corollaire 2. - Si $K=n$ alors $s_{2}=n$; ainsi, pour tout $s \in \mathbb{N}$ tel que $s>n$, la solution de $(\Delta+K) \varphi=\rho^{s}$ qui s'annule sur $\partial B$ est dans $\Lambda_{0}^{k}$ si et seulement si $k \leq n$.

Corollaire 3. - Si $K=2 n+2$ alors $s_{2}=n+1$; ainsi, pour tout $s \in \mathbb{N}$ tel que $s>n+1$, la solution de $(\Delta+K) \varphi=\rho^{s}$ qui s'annule sur $\partial B$ est dans $\Lambda_{0}^{k}$ si et seulement si $k \leq n+1$.

Corollaire 4. - S'il existe $\epsilon>0$ et un réel $t>s_{2}$ tels que $\sigma \geq \epsilon \rho^{t}$, et si $v$ est solution nulle sur $\partial B$ de $(\Delta+K) v \geq \sigma$, alors $v$ ne peut être dans $\Lambda_{0}^{k}$ si $k>s_{2}$.

Preuve du corollaire 4. - Prenons $s=s_{2}+E\left(t-s_{2}+1\right)$; alors $\rho^{t} \geq \rho^{s}$ donc, par le principe du maximum, $v \geq \epsilon \varphi$.

\section{Appendice}

\subsection{Une estimation non-linéaire.}

Lemme 3. - Soit $v$ dans $\Lambda_{\ell, \alpha}^{s}(B)$, avec $s \geq 0,\|v\|_{\ell, \alpha}^{s} \leq K,-a \leq v \leq b$ où $a, b \in \mathbb{R}^{+}$. Si $\Psi$ appartient ̀̀ $C^{\ell+1}([-a, b])$ avec $\Psi(0)=0$, alors $u=(\Psi \circ v)$ appartient à $\Lambda_{\ell, \alpha}^{s}(B)$ et $\|\Psi \circ v\|_{\ell, \alpha}^{s}$ est majorée par une constante qui ne dépend de $v$ qu'à travers $K$, a et $b$ (nous noterons ici $C(K, a, b)$ de telles constantes).

Preuve. - Rappelons que $d_{x}=\operatorname{dist}(x, \partial B)=1-|x|$, et que

$$
\begin{aligned}
\|u\|_{\ell, \alpha}^{s}= & \sum_{|\gamma| \leq \ell} \sup _{x \in B}\left[d_{x}^{-s+|\gamma|}\left|\partial^{\gamma} u(x)\right|\right] \\
& +\sum_{|\gamma|=\ell} \sup _{\substack{x, y \in B \\
x \neq y}} \min \left(d_{x}^{-s+\ell+\alpha}, d_{y}^{-s+\ell+\alpha}\right) \frac{\left|\partial^{\gamma} u(x)-\partial^{\gamma} u(y)\right|}{|x-y|^{\alpha}} \\
= & \sum_{|\gamma| \leq \ell}\left\|\partial^{\gamma} u\right\|_{0}^{s-|\gamma|} \\
& \quad+\sum_{|\gamma|=\ell} \sup _{\substack{x, y \in B \\
x \neq y}} \min \left(d_{x}^{-s+\ell+\alpha}, d_{y}^{-s+\ell+\alpha}\right) \frac{\left|\partial^{\gamma} u(x)-\partial^{\gamma} u(y)\right|}{|x-y|^{\alpha}} .
\end{aligned}
$$

a) Supposons $|\gamma|=0$. On a alors facilement :

$$
\begin{aligned}
\sup _{x \in B} d_{x}^{-s}|u(x)| & =\sup _{x \in B} d_{x}^{-s}|v(x)| \frac{|\Psi \circ v(x)|}{|v(x)|} \\
& \leq \sup _{[-a, b]}\left|\frac{\Psi(t)}{t}\right| \sup _{x \in B} d_{x}^{-s}|v(x)|=C(K, a, b)\|v\|_{0}^{s} .
\end{aligned}
$$

TOME $125-1997-\mathrm{N}^{\circ} 3$ 
b) Supposons $1 \leq|\gamma| \leq \ell$. Il est facile de s'assurer par récurrence (c'est un cas particulier de la formule de Faa di Bruno) que $\partial^{\gamma} u$ est une somme de termes de la forme

$$
\prod_{i \in I}\left(\partial^{\mu_{i}} v\right)^{r_{i}}\left(\Psi^{(j)} \circ v\right)
$$

où $I$ est fini non vide, $r_{i} \in \mathbb{N}, 1 \leq j \leq|\gamma|, 1 \leq\left|\mu_{i}\right| \leq|\gamma|$,

$$
\sum_{i \in I}\left|\mu_{i}\right| r_{i}=|\gamma| \quad \text { et } \quad \Psi^{(j)}=\frac{\mathrm{d}^{j} \Psi}{\mathrm{d} t^{j}}
$$

Comme $\left\|\partial^{\mu_{i}} v\right\|_{0}^{s-\left|\mu_{i}\right|} \leq C(K, a, b)$, et comme

$$
\left\|\Psi^{(j)} \circ v\right\|_{0}^{0} \leq \sup _{t \in[-a, b]}\left|\Psi^{(j)}(t)\right| \leq C(K, a, b)
$$

d'après [GL, prop. 3.3, p. 208], le terme ci-dessus est dans

$$
\Lambda_{0}^{\sum r_{i}\left(s-\left|\mu_{i}\right|\right)}=\Lambda_{0}^{\left.\sum r_{i} s-\sum r_{i}\left|\mu_{i}\right|\right)}=\Lambda_{0}^{\left(\sum r_{i}\right) s-|\gamma|},
$$

les sommations se faisant pour $i \in I$, et puisque $\sum_{i \in I} r_{i} \geq 1$, ce terme est donc au moins dans $\Lambda_{0}^{s-|\gamma|}$ et majoré en norme \|\|$_{0}^{s-|\gamma|} \operatorname{par} C(K, a, b)$. Ainsi

$$
\left\|\partial^{\gamma} u\right\|_{0}^{s-|\gamma|} \leq C(K, a, b) .
$$

c) Supposons $|\gamma|=\ell$. En raisonnant comme précédemment et en notant

$$
w=\prod_{i \in I}\left(\partial^{\mu_{i}} v\right)^{r_{i}}
$$

$w$ est au moins dans $\Lambda_{0, \alpha}^{s-\ell}$ et $\|w\|_{0, \alpha}^{s-\ell} \leq C(K, a, b)$. Maintenant,

$$
\min \left(d_{x}^{-s+\ell+\alpha}, d_{y}^{-s+\ell+\alpha}\right) \frac{\left|\partial^{\gamma} u(x)-\partial^{\gamma} u(y)\right|}{|x-y|^{\alpha}}
$$

est majoré par une somme de termes de la forme :

$$
\begin{aligned}
& \min \left(d_{x}^{-s+\ell+\alpha}, d_{y}^{-s+\ell+\alpha}\right) \frac{\left|w(x) \Psi^{(j)}(v(x))-w(y) \Psi^{(j)}(v(y))\right|}{|x-y|^{\alpha}} \\
& \quad \leq(\mathrm{i})+(\mathrm{ii})
\end{aligned}
$$


avec

$$
\begin{aligned}
& \text { (i) }=\min \left(d_{x}^{-s+\ell+\alpha}, d_{y}^{-s+\ell+\alpha}\right) \frac{\left|w(x) \Psi^{(j)}(v(x))-w(y) \Psi^{(j)}(v(x))\right|}{|x-y|^{\alpha}}, \\
& \text { (ii) }=\min \left(d_{x}^{-s+\ell+\alpha}, d_{y}^{-s+\ell+\alpha}\right) \frac{\left|w(y) \Psi^{(j)}(v(x))-w(y) \Psi^{(j)}(v(y))\right|}{|x-y|^{\alpha}} .
\end{aligned}
$$

D'une part,

$$
\text { (i) } \begin{aligned}
& \leq \min \left(d_{x}^{-s+\ell+\alpha}, d_{y}^{-s+\ell+\alpha}\right) \frac{|w(x)-w(y)|}{|x-y|^{\alpha}}\left|\Psi^{(j)}(v(x))\right| \\
& \leq\|w\|_{0, \alpha}^{s-\ell} \sup _{t \in[-a, b]}\left|\Psi^{(j)}(t)\right| \leq C(K, a, b) .
\end{aligned}
$$

Compte-tenu du théorème des accroissements finis (appliqué à $\Psi^{(j)}$ ), on a d'autre part :

$$
\begin{aligned}
\text { (ii) } \leq \min \left(d_{x}^{-s+\ell+\alpha}, d_{y}^{-s+\ell+\alpha}\right) \frac{|v(x)-v(y)|}{|x-y|^{\alpha}} & \times \frac{\left|\Psi^{(j)}(v(x))-\Psi^{(j)}(v(y))\right|}{|v(x)-v(y)|}|w(y)| \\
& \leq \min \left(d_{x}^{-s+\ell+\alpha}, d_{y}^{-s+\ell+\alpha}\right) \frac{|v(x)-v(y)|}{|x-y|^{\alpha}}|w(y)| \sup _{t \in[-a, b]}\left|\Psi^{(j+1)}(t)\right| .
\end{aligned}
$$

Distinguons deux cas :

- $\mathrm{Si}-s+\ell \geq 0$, alors

$$
\begin{aligned}
\min \left(d_{x}^{-s+\ell+\alpha}, d_{y}^{-s+\ell+\alpha}\right) & =\left[\min \left(d_{x}, d_{y}\right)\right]^{-s+\ell+\alpha} \\
& \leq d_{y}^{-s+\ell}\left[\min \left(d_{x}, d_{y}\right)\right]^{\alpha}=d_{y}^{-s+\ell}\left[\min \left(d_{x}^{\alpha}, d_{y}^{\alpha}\right)\right]
\end{aligned}
$$

et donc

$$
\text { (ii) } \leq\|v\|_{0, \alpha}^{0}\|w\|_{0}^{s-\ell} \sup _{t \in[-a, b]}\left|\Psi^{(j+1)}(t)\right| \leq C(K, a, b) \text {. }
$$

- $\mathrm{Si}-s+\ell<0$, alors $d_{y}^{-s+\ell} \geq 1$; ainsi $|w(y)| \leq d_{y}^{-s+\ell}|w(y)| \leq\|w\|_{0}^{s-\ell}$ et

$$
\text { (ii) } \leq\|v\|_{0, \alpha}^{s-\ell}\|w\|_{0}^{s-\ell} \sup _{t \in[-a, b]}\left|\Psi^{(j+1)}(t)\right| \leq C(K, a, b) \text {. }
$$

6.2. Compacité des injections $\Lambda_{k, \alpha}^{s} \rightarrow \Lambda_{k, \beta}^{t}$ lorsque $s>t$ et $\mathbf{0}<\boldsymbol{\beta}<\boldsymbol{\alpha}<\mathbf{1}$.

Pour tout entier positif $j$, posons $B_{j}=B\left(0,1-2^{-j}\right)$. 
Lemme 4. - Pour tout entier positif $k$, il existe une suite de fonctions $\left\{\varphi_{j}\right\}_{j>0}$ possédant les propriétés suivantes:

$$
\varphi_{j}= \begin{cases}1 & \text { sur } B_{j} \\ 0 & \text { sur } B \backslash B_{j+1}\end{cases}
$$

avec $\varphi_{j} \in C_{\mathrm{loc}}^{k+1}(B)$ et, si on note $d_{j}=\operatorname{dist}\left(B_{j}, \partial B\right)=2^{-j}$ alors, pour tout $x \in B$ et tout $\gamma$ tel que $|\gamma| \leq k+1$, on $a$ :

$$
\left|\partial \varphi_{j}(x)\right| \leq \frac{C(|\gamma|, k)}{d_{j}^{|\gamma|}}
$$

Preuve. - Notons que la suite $\left\{B_{j}\right\}_{j>0}$ vérifie $: \bar{B}_{j} \subset \subset B_{j+1} \subset B$ avec

$$
\operatorname{dist}\left(\bar{B}_{j}, \partial B_{j+1}\right)=d_{j}-d_{j+1}=\frac{1}{2} d_{j}=d_{j+1} .
$$

Nous allons construire $\varphi_{j}$ par étapes.

a) Pour $u \in[0,1]$, soit $f(u)=\int_{u}^{1} t^{k+1}(1-t)^{k+1} \mathrm{~d} t$. Alors $f(0)>0$, $f(1)=0$ et pour $i=1, \ldots, k+1$, on a :

$$
f^{(i)}(0)=f^{(i)}(1)=0 \quad \text { et } \quad\left|f^{(i)}(u)\right| \leq C(i, k) .
$$
on $\mathrm{a}$ :

b) Pour $u \in\left[0, \frac{1}{2} d_{j}\right]$, soit $g_{j}(u)=\frac{1}{f(0)} f\left(\frac{2 u}{d_{j}}\right)$. Alors, pour $i \leq k+1$,

$$
\left|g_{j}^{(i)}(u)\right| \leq \frac{C(i, k)}{\left(d_{j}\right)^{i}}
$$

c) Soit

$$
h_{j}(u)= \begin{cases}1 & \text { sur }\left[0,1-d_{j}\right] \\ g_{j}\left(u-1+d_{j}\right) & \text { sur }\left[1-d_{j}, 1-d_{j+1}\right] \\ 0 & \text { sur }\left[1-d_{j+1}, 1\right]\end{cases}
$$

Les dérivées de $h_{j}$ se recollent bien et sont bornées comme précédemment : on a $h_{j} \in C^{k+1}([0,1])$ et $\left|h_{j}^{(i)}\right| \leq \frac{C(i, k)}{\left(d_{j}\right)^{i}}$ pour $i \in\{1, . ., k+1\}$.

d) Soit $\varphi_{j}(x)=h_{j}(|x|)$ pour $x \in \mathbb{R}^{n}$ et $j \geq 1$. Les dérivées de $x \mapsto|x|$ sont bornées sur $\overline{B \backslash B_{1}}$; or $\partial^{\gamma} \varphi_{j}$ ne fait intervenir que des dérivées d'ordre au plus $|\gamma|$ de $h_{j}$ sur $\left[1-d_{j}, 1-d_{j+1}\right]$, et de $|x|$ sur $\overline{B_{j+1} \backslash B_{j}} \subset \overline{B \backslash B_{1}}$. Comme $0<d_{j}<1$, pour tout $m \leq|\gamma|$, on a $d_{j}^{-|\gamma|} \geq d_{j}^{-m}$, donc $\left|\partial^{\gamma} \varphi_{j}(x)\right| \leq C(|\gamma|, k) / d_{j}^{|\gamma|}$. 
Corollaire 5. - Pour tout $k \in \mathbb{N}$ et tout $j \geq 1$, on a $\varphi_{j} \in \Lambda_{k+1}^{s}$ et $\left\|\varphi_{j}\right\|_{k+1}^{0} \leq C(k, \hat{\jmath})$.

Nota BenE. - L'indépendance de l'estimation sur $\left\|\varphi_{j}\right\|_{k+1}^{0}$ par rapport à $j$ est essentielle. Elle n'a plus lieu pour $\left\|\varphi_{j}\right\|_{k+1}^{s}$ avec $s \neq 0$.

Preuve. - Si $|\gamma|=0$, on a $\left|\varphi_{j}\right| \leq 1$. Et pour tout $\gamma$ tel que $1 \leq|\gamma| \leq k+1$, on a $\partial^{\gamma} \varphi_{j}=0$ sauf sur $d_{j+1} \leq d \leq d_{j}$; donc

$$
d^{|\gamma|}\left|\partial^{\gamma} \varphi_{j}\right| \leq d_{j}^{|\gamma|}\left|\partial^{\gamma} \varphi_{j}\right| \leq d_{j}^{|\gamma|} \frac{C(|\gamma|, k)}{d_{j}^{|\gamma|}} \leq C(|\gamma|, k) .
$$

Pour $\Omega$ ouvert tel que $\bar{\Omega} \subset \subset B$, posons :

$$
C_{0}^{k, \beta}(\bar{\Omega}):=\left\{u \in C^{k, \beta}(\bar{\Omega}) ; \forall|\gamma| \leq k, \partial^{\gamma} u=0 \text { sur } \partial \Omega\right\} .
$$

L'ensemble $C_{0}^{k, \beta}(\bar{\Omega})$ est un sous-espace de Banach fermé de $C^{k, \beta}(\bar{\Omega})$. Pour $u \in C_{0}^{k, \beta}(\bar{\Omega})$, soit $\widetilde{u}$ l'extension canonique de $u$ à $B$ par zéro hors de $\Omega$ : il est immédiat de vérifier que $\widetilde{u}$ appartient à $C^{k, \beta}(\bar{B})$. et

Lemme 5. - Pour tout $u \in C_{0}^{k, \beta}(\bar{\Omega})$, pour tout $t \in \mathbb{R}$, on a $\widetilde{u} \in \Lambda_{k, \beta}^{t}(B)$

$$
\|\widetilde{u}\|_{k, \beta}^{t}<C(\operatorname{dist}(\partial \Omega, \partial B), t, k, \beta)\|u\|_{k, \beta, \bar{\Omega}} .
$$

Autrement dit, l'extension $u \mapsto \widetilde{u}$ fournit une inclusion continue

$$
C_{0}^{k, \beta}(\bar{\Omega}) \longrightarrow \Lambda_{k, \beta}^{t}(B)
$$

Preuve. - Soit $u \in C_{0}^{k, \beta}(\bar{\Omega})$; nous voulons une majoration convenable de

$$
\begin{aligned}
\|\widetilde{u}\|_{k, \beta, B}^{t} & =\sum_{|\gamma| \leq k} \sup _{x \in B}\left[d_{x}^{-t+|\gamma|}\left|\partial^{\gamma} \widetilde{u}(x)\right|\right] \\
& +\sum_{|\gamma|=k} \sup _{\substack{x, y \in B \\
x \neq y}} \min \left(d_{x}^{-t+k+\beta}, d_{y}^{-t+k+\beta}\right) \frac{\left|\partial^{\gamma} \widetilde{u}(x)-\partial^{\gamma} \widetilde{u}(y)\right|}{|x-y|^{\beta}} .
\end{aligned}
$$

Déjà, pour tout $|\gamma| \leq k$, on a

$$
\begin{aligned}
\sup _{B} d^{-t+|\gamma|}\left|\partial^{\gamma} \widetilde{u}\right| & =\sup _{\bar{\Omega}} d^{-t+|\gamma|}\left|\partial^{\gamma} u\right| \\
& \leq C(\operatorname{dist}(\partial \Omega, \partial B), t,|\gamma|)\|u\|_{k, \beta, \bar{\Omega}}
\end{aligned}
$$

car sur $\bar{\Omega}$, on a $0<\operatorname{dist}(\partial \Omega, \partial B) \leq d \leq 1$ 
Ensuite, si $|\gamma|=k$, notons

$$
U_{x, y}^{t}=\min \left(d_{x}^{-t+k+\beta}, d_{y}^{-t+k+\beta}\right) \frac{\left|\partial^{\gamma} \widetilde{u}(x)-\partial^{\gamma} \widetilde{u}(y)\right|}{|x-y|^{\beta}}
$$

Si $x, y$ sont dans $B \backslash \Omega$, alors $U_{x, y}^{t}=0$. Si $x, y$ sont dans $\Omega$, alors

$$
\begin{aligned}
U_{x, y}^{t} & =\min \left(d_{x}^{-t+k+\beta}, d_{y}^{-t+k+\beta}\right) \frac{\left|\partial^{\gamma} u(x)-\partial^{\gamma} u(y)\right|}{|x-y|^{\beta}} \\
& \leq C(\operatorname{dist}(\partial \Omega, \partial B), t, k, \beta)\|u\|_{k, \beta, \bar{\Omega}} .
\end{aligned}
$$

Enfin, si $x$ est dans $\Omega$ et $y$ dans $B \backslash \Omega$, il faut distinguer encore deux sous-cas.

- Premier cas : $|x-y| \leq \frac{1}{2} d_{x}$. Soit $z$ un point d'intersection du segment $[x, y]$ avec $\partial \Omega$; alors $\partial^{\gamma} u(z)=\partial^{\gamma} u(y)=0$, donc

$$
\begin{aligned}
U_{x, y}^{t} & =\min \left(d_{x}^{-t+k+\beta}, d_{y}^{-t+k+\beta}\right) \frac{\left|\partial^{\gamma} u(x)-\partial^{\gamma} u(z)\right|}{|x-y|^{\beta}} \\
& \leq \min \left(d_{x}^{-t+k+\beta}, d_{y}^{-t+k+\beta}\right) \frac{\left|\partial^{\gamma} u(x)-\partial^{\gamma} u(z)\right|}{|x-z|^{\beta}} \\
& \leq \mathrm{C}^{\mathrm{te}}\left(d_{x}^{-t+k+\beta}, d_{y}^{-t+k+\beta}\right)\|u\|_{k, \beta, \bar{\Omega}} .
\end{aligned}
$$

Or $\operatorname{dist}(\partial \Omega, \partial B) \leq d_{x} \leq 1$ et, pour tout $y \in B\left(x, \frac{1}{2} d_{x}\right)$, on a

$$
\frac{1}{2} \operatorname{dist}(\partial \Omega, \partial B) \leq \frac{1}{2} d_{x} \leq d_{y} \leq \frac{3}{2} d_{x} \leq \frac{3}{2}
$$

donc on a bien

$$
U_{x, y}^{t} \leq C(\operatorname{dist}(\partial \Omega, \partial B), t, k, \beta)\|u\|_{k, \beta, \bar{\Omega}}
$$

- Deuxième cas : $|x-y|>\frac{1}{2} d_{x}$. Dans ce cas

$$
U_{x, y}^{t} \leq d_{x}^{-t+k+\beta} \frac{\left|\partial^{\gamma} u(x)\right|}{d_{x}^{\beta} / 2^{\beta}}=2^{\beta} d_{x}^{-t+k}\left|\partial^{\gamma} u(x)\right| \leq 2^{\beta} d_{x}^{-t+k} \mathrm{C}^{\mathrm{te}}\|u\|_{k, \bar{\Omega}}
$$

et comme on a toujours $\operatorname{dist}(\partial \Omega, \partial B) \leq d_{x} \leq 1$, finalement

$$
U_{x, y}^{t} \leq C(\operatorname{dist}(\partial \Omega, \partial B), t, k, \beta)\|u\|_{k, \beta, \bar{\Omega}} \cdot \quad \square
$$


Lemme 6. - Soient $u \in \Lambda_{k, \alpha}^{s}$ et $C$ une constante telle que $\|u\|_{k, \alpha}^{s} \leq C$. Alors, pour tout $t<s$, on $a$ :

$$
\left\|u\left(1-\varphi_{j}\right)\right\|_{k, \alpha}^{t} \leq \mathrm{C}^{\mathrm{te}}(C, k, \alpha, s, t) d_{j}^{s-t} .
$$

On a donc en particulier

$$
\lim _{j \rightarrow \infty}\left\|u\left(1-\varphi_{j}\right)\right\|_{k, \alpha}^{t}=0 .
$$

Preuve. - Rappelons que

$$
\begin{aligned}
\left\|u\left(1-\varphi_{j}\right)\right\|_{k, \alpha}^{t} & =\sum_{|\gamma| \leq k} \sup _{x \in B}\left[d_{x}^{-t+|\gamma|}\left|\partial^{\gamma}\left(u\left(1-\varphi_{j}\right)\right)(x)\right|\right] \\
+ & \sum_{|\gamma|=k} \sup _{\substack{x, y \in B \\
x \neq y}} \min \left(d_{x}^{-t+k+\alpha}, d_{y}^{-t+k+\alpha}\right) \\
& \times \frac{\left|\partial^{\gamma}\left(u\left(1-\varphi_{j}\right)\right)(x)-\partial^{\gamma}\left(u\left(1-\varphi_{j}\right)\right)(y)\right|}{|x-y|^{\alpha}} .
\end{aligned}
$$

Tout d'abord, pour $|\gamma| \leq k$ :

$$
\partial^{\gamma}\left(u\left(1-\varphi_{j}\right)\right)=\sum_{\mu+\nu=\gamma} \mathrm{C}^{\mathrm{te}}(|\mu|,|\nu|) \partial^{\mu} u \partial^{\nu}\left(1-\varphi_{j}\right),
$$

où $\gamma=\mu+\nu$ signifie que le couple de multi-indices $(\mu, \nu)$ est une partition du multi-indice $\gamma$. Or avec $\gamma=\mu+\nu$,

$$
\begin{aligned}
\sup _{B} d^{-t+|\gamma|}\left|\partial^{\mu} u \partial^{\nu}\left(1-\varphi_{j}\right)\right| \\
=\sup _{B \backslash B_{j}} d^{-t+|\gamma|}\left|\partial^{\mu} u \partial^{\nu}\left(1-\varphi_{j}\right)\right| \\
=\sup _{B \backslash B_{j}} d^{s-t+|\nu|} d^{-s+|\mu|}\left|\partial^{\mu} u \partial^{\nu}\left(1-\varphi_{j}\right)\right| \\
\leq d_{j}^{s-t+|\nu|} \sup _{B \backslash B_{j}} d^{-s+|\mu|}\left|\partial^{\mu} u \partial^{\nu}\left(1-\varphi_{j}\right)\right| \\
\leq d_{j}^{s-t+|\nu|} \sup _{B \backslash B_{j}}\left|\partial^{\nu}\left(1-\varphi_{j}\right)\right| \sup _{B \backslash B_{j}} d^{-s+|\mu|}\left|\partial^{\mu} u\right| \\
\leq d_{j}^{s-t+|\nu|} \frac{\mathrm{C}^{\mathrm{te}}(|\nu|, C)}{d_{j}^{|\nu|}\left\|\partial^{\mu} u\right\|_{0,0}^{s-|\mu|}} \\
\leq \mathrm{C}^{\mathrm{te}}(|\nu|, C)\|u\|_{k, \alpha}^{s} d_{j}^{s-t} .
\end{aligned}
$$

Maintenant, si $|\gamma|=k$, notons

$$
V_{x, y}^{t}=\min \left(d_{x}^{-t+k+\alpha}, d_{y}^{-t+k+\alpha}\right) \frac{\left|\partial^{\gamma}\left(u\left(1-\varphi_{j}\right)\right)(x)-\partial^{\gamma}\left(u\left(1-\varphi_{j}\right)\right)(y)\right|}{|x-y|^{\alpha}} .
$$


Si $x, y$ sont dans $B_{j}$, on a $V_{x, y}^{t}=0$.

Si $x, y$ sont dans $B \backslash B_{j}$, comme sur $B \backslash B_{j}$ on a

$$
d^{-t+k+\alpha}=d^{s-t} d^{-s+k+\alpha} \leq d_{j}^{s-t} d^{-s+k+\alpha},
$$

alors

$$
V_{x, y}^{t} \leq d_{j}^{s-t} V_{x, y}^{s} \leq d_{j}^{s-t}\left\|u\left(1-\varphi_{j}\right)\right\|_{k, \alpha}^{s} \leq d_{j}^{s-t}\|u\|_{k, \alpha}^{s}\left\|1-\varphi_{j}\right\|_{k+1}^{0},
$$

la dernière égalité ayant lieu d'après [GL, p. 208].

Si $y$ est dans $\overline{B_{j}}$ et $x$ dans $\overline{B \backslash B_{j+1}}$, alors $|x-y| \geq d_{j+1}$ et

$$
\begin{aligned}
V_{x, y}^{t} & =\min \left(d_{x}^{-t+k+\alpha}, d_{y}^{-t+k+\alpha}\right) \frac{\left|\partial^{\gamma} u(x)\right|}{|x-y|^{\alpha}} \leq d_{x}^{-t+k+\alpha} \frac{\left|\partial^{\gamma} u(x)\right|}{d_{j+1}^{\alpha}} \\
& \leq d_{x}^{s-t+\alpha} d_{x}^{-s+k} \frac{\left|\partial^{\gamma} u(x)\right|}{d_{j+1}^{\alpha}} \leq d_{j+1}^{s-t+\alpha} d_{x}^{-s+k} \frac{\left|\partial^{\gamma} u(x)\right|}{d_{j+1}^{\alpha}} \\
& \leq \frac{d_{j}^{s-t}}{2^{s-t}} \mathrm{C}^{\mathrm{te}}\|u\|_{k}^{s} .
\end{aligned}
$$

Si $y$ appartient à $\overline{B_{j-1}}$ et si $x$ appartient à $\overline{B \backslash B_{j}}$, on a de même $V_{x, y}^{t} \leq d_{j}^{s-t} \mathrm{C}^{\mathrm{te}}\|u\|_{k}^{s}$.

Enfin, si $y$ appartient à $B_{j} \backslash B_{j-1}$ et si $x$ appartient à $B_{j+1} \backslash B_{j}$, distinguons deux cas :

- $\mathrm{Si}-s+k+\alpha \geq 0$, alors $-t+k+\alpha \geq 0$ et

$$
\begin{aligned}
\min \left(d_{x}^{-t+k+\alpha}, d_{y}^{-t+k+\alpha}\right) & =d_{x}^{-t+k+\alpha}=d_{x}^{s-t} d_{x}^{-s+k+\alpha} \\
& \leq d_{j}^{s-t} d_{x}^{-s+k+\alpha} \\
& =d_{j}^{s-t} \min \left(d_{x}^{-s+k+\alpha}, d_{y}^{-s+k+\alpha}\right) .
\end{aligned}
$$

- $\mathrm{Si}-s+k+\alpha<0$, alors

$$
\begin{aligned}
\min \left(d_{x}^{-t+k+\alpha}, d_{y}^{-t+k+\alpha}\right) & \leq d_{y}^{-t+k+\alpha}=d_{y}^{s-t} d_{y}^{-s+k+\alpha} \\
& \leq d_{j-1}^{s-t} d_{y}^{-s+k+\alpha} \\
& =2^{s-t} d_{j}^{s-t} \min \left(d_{x}^{-s+k+\alpha}, d_{y}^{-s+k+\alpha}\right) .
\end{aligned}
$$

Ainsi dans les deux cas,

$$
V_{x, y}^{t} \leq d_{j}^{s-t} \mathrm{C}^{\mathrm{te}}\left\|u\left(1-\varphi_{j}\right)\right\|_{k, \alpha}^{s} \leq d_{j}^{s-t} \mathrm{C}^{\mathrm{te}}\|u\|_{k, \alpha}^{s} \cdot\left\|1-\varphi_{j}\right\|_{k+1}^{0} .
$$

Il ne nous reste plus qu'à remarquer que, tout comme dans le corollaire 5 du paragraphe 6 , on a $\left\|1-\varphi_{j}\right\|_{k+1}^{0} \leq C(k, \hat{\jmath})$ pour achever la preuve du lemme 6 . 
Proposition 8. - Pour tout $k \in \mathbb{N}$, pour tous $s>t \in \mathbb{R}$ et pour tout $0<\beta<\alpha<1$, l'injection $\Lambda_{k, \alpha}^{s} \rightarrow \Lambda_{k, \beta}^{t}$ est compacte.

Preuve. - Dans tout ce qui suit, il sera commode de conserver abusivement la même notation pour une suite et diverses sous-suites extraites.

Soit $\left\{u_{p}\right\}_{p \in \mathbb{N}}$ suite de $\Lambda_{k, \alpha}^{s}$ uniformément bornée en norme $\Lambda_{k, \alpha}^{s}$ par une constante $C$. On veut en extraire une sous-suite qui converge dans $\Lambda_{k, \beta}^{t}$. Il suffit pour cela qu'elle soit de Cauchy, i.e. telle que :

$$
\forall \epsilon>0, \exists N, \forall p, q>N, \quad\left\|u_{p}-u_{q}\right\|_{k, \beta}^{t}<\epsilon .
$$

Pour chaque $j \geq 1$ nous écrirons :

$$
u_{p}=u_{p} \varphi_{j}+u_{p}\left(1-\varphi_{j}\right) .
$$

La suite $\left\{u_{p} \varphi_{1}\right\}_{p}$ est bornée dans $C^{k, \alpha}\left(\bar{B}_{2}\right)$; donc il existe une soussuite de $\left\{u_{p}\right\}_{p}$ telle que $\left\{u_{p} \varphi_{1}\right\}_{p}$ converge vers $v_{1} \in C^{k, \alpha}\left(\bar{B}_{2}\right)$.

En repartant de cette sous-suite, on considère $\left\{u_{p} \varphi_{2}\right\}_{p}$ qui est une suite bornée de $C^{k, \alpha}\left(\bar{B}_{3}\right)$ donc il existe une sous-suite de $\left\{u_{p}\right\}_{p}$ telle que $\left\{u_{p} \varphi_{2}\right\}_{p}$ converge vers $v_{2} \in C^{k, \alpha}\left(\bar{B}_{3}\right)$.

Une fois itérée cette procédure sur $\left\{u_{p} \varphi_{j}\right\}_{p}$ pour tout $j \geq 1$, nous extrayons de $\left\{u_{p}\right\}_{p}$ une sous-suite diagonale qui possède la propriété suivante : pour tout $j \geq 1$, il existe $v_{j} \in C^{k, \beta}\left(\bar{B}_{j+1}\right)$ telle que $\left\{u_{p} \varphi_{j}\right\}_{p}$ converge vers $v_{j}$ dans $C^{k, \beta}\left(\bar{B}_{j+1}\right)$; comme $u_{p} \varphi_{j}$ est à support dans $B_{j+1}$, on voit que $v_{j}$ appartient à $C_{0}^{k, \beta}$. D'après le lemme 5 nous aurons donc $\lim _{p \rightarrow \infty} u_{p} \varphi_{j}=\widetilde{v_{j}}$ dans $\Lambda_{k, \beta}^{t}(B)$. Ainsi :

$$
\forall j \geq 1, \forall \epsilon>0, \exists N \in \mathbb{N}, \forall p, q>N, \quad\left\|u_{p} \varphi_{j}-u_{q} \varphi\right\|_{k, \beta}^{t}<\frac{1}{2} \epsilon .
$$

D'après [GL, p. 208] et d'après le lemme 6, comme $\left\|u_{p}-u_{q}\right\|_{k, \alpha}^{s} \leq 2 C$,

$$
\begin{aligned}
\left\|\left(u_{p}-u_{q}\right)\left(1-\varphi_{j}\right)\right\|_{k, \beta}^{t} & \leq \mathrm{C}^{\mathrm{te}}(\alpha, \beta)\left\|\left(u_{p}-u_{q}\right)\left(1-\varphi_{j}\right)\right\|_{k, \alpha}^{t} \\
& \leq \mathrm{C}^{\mathrm{te}}(C, k, \alpha, \beta, s, t) d_{j}^{s-t}
\end{aligned}
$$

d'où

$$
\forall \epsilon>0, \exists j, \forall p, q, \quad\left\|\left(u_{p}-u_{q}\right)\left(1-\varphi_{j}\right)\right\|_{k, \beta}^{t} \leq \frac{1}{2} \epsilon .
$$

Un tel $j$ étant fixé,

$$
\exists N, \forall p, q>N, \quad\left\|u_{p} \varphi_{j}-u_{q} \varphi_{j}\right\|_{k, \beta}^{t} \leq \frac{1}{2} \epsilon,
$$

soit finalement :

$$
\left\|u_{p}-u_{q}\right\|_{k, \beta}^{t} \leq\left\|\left(u_{p}-u_{q}\right) \varphi_{j}\right\|_{k, \beta}^{t}+\left\|\left(u_{p}-u_{q}\right)\left(1-\varphi_{j}\right)\right\|_{k, \beta}^{t}<\epsilon .
$$

TOME $125-1997-\mathrm{N}^{\circ} 3$ 
Lemme 7. - Soit $\left\{u_{i}\right\}_{i}$ une suite de $\Lambda_{k, \alpha}^{s}$ telle que pour tout $i$, on ait $\left\|u_{i}\right\|_{k, \alpha}^{s} \leq C$ et $\left\{u_{i}\right\}_{i}$ converge vers $u$ dans $\Lambda_{k, \beta}^{t}$, avec $s>t$ et $0<\beta<\alpha<1$. Alors $u$ appartient à $\Lambda_{k, \alpha}^{s}$ et

$$
\|u\|_{k, \alpha}^{s} \leq \mathrm{C}^{\mathrm{te}}(k, n) C .
$$

Preuve. - On rappelle que

$$
\begin{aligned}
\left\|u_{i}\right\|_{k, \alpha}^{s}= & \sum_{|\gamma| \leq k} \sup _{x \in B}\left[d_{x}^{-s+|\gamma|}\left|\partial^{\gamma} u_{i}(x)\right|\right] \\
& +\sum_{|\gamma|=k} \sup _{\substack{x, y \in B \\
x \neq y}} \min \left(d_{x}^{-s+k+\alpha}, d_{y}^{-s+k+\alpha}\right) \frac{\left|\partial^{\gamma} u_{i}(x)-\partial^{\gamma} u_{i}(y)\right|}{|x-y|^{\alpha}} .
\end{aligned}
$$

Si $|\gamma| \leq k$, alors en tout point $x \in B$, on a :

$$
\begin{aligned}
d_{x}^{-s+|\gamma|}\left|\partial^{\gamma} u(x)\right| & \leq d_{x}^{-s+|\gamma|}\left|\partial^{\gamma} u_{i}(x)\right|+d_{x}^{-s+|\gamma|}\left|\partial^{\gamma} u(x)-\partial^{\gamma} u_{i}(x)\right| \\
& \leq C+d_{x}^{-s+t} d_{x}^{-t+|\gamma|}\left|\partial^{\gamma} u(x)-\partial^{\gamma} u_{i}(x)\right| \\
& \leq C+d_{x}^{t-s}\left\|u-u_{i}\right\|_{k, \beta}^{t} .
\end{aligned}
$$

Comme $\lim _{i \rightarrow \infty}\left\|u-u_{i}\right\|_{k, \beta}^{t}=0$, on en déduit (à $x$ fixé et $i \rightarrow \infty$ ) :

$$
d_{x}^{-s+|\gamma|}\left|\partial^{\gamma} u(x)\right| \leq C,
$$

et par suite

$$
\sup _{x \in B} d_{x}^{-s+|\gamma|}\left|\partial^{\gamma} u(x)\right| \leq C .
$$

Si $|\gamma|=k$, alors pour tout couple $(x, y)$ de $B$, avec $x \neq y$, en supposant $\min \left(d_{x}^{-s+k+\alpha}, d_{y}^{-s+k+\alpha}\right)=d_{x}^{-s+k+\alpha}$ (quitte à inverser $x$ et $y$ ), on a :

$$
\begin{aligned}
d_{x}^{-s+k+\alpha} \frac{\left|\partial^{\gamma} u(x)-\partial^{\gamma} u(y)\right|}{|x-y|^{\alpha}} \leq & d_{x}^{-s+k+\alpha} \frac{\left|\partial^{\gamma} u(x)-\partial^{\gamma} u_{i}(x)\right|}{|x-y|^{\alpha}} \\
& +d_{x}^{-s+k+\alpha} \frac{\left|\partial^{\gamma} u_{i}(x)-\partial^{\gamma} u_{i}(y)\right|}{|x-y|^{\alpha}} \\
& +d_{x}^{-s+k+\alpha} \frac{\left|\partial^{\gamma} u_{i}(y)-\partial^{\gamma} u(y)\right|}{|x-y|^{\alpha}} \\
\leq & \frac{d_{x}^{t-s+\alpha}}{|x-y|^{\alpha}}\left[d_{x}^{-t+k} \cdot\left|\partial^{\gamma} u(x)-\partial^{\gamma} u_{i}(x)\right|\right]+C \\
\quad+\frac{1}{|x-y|^{\alpha}} \frac{d_{x}^{-s+k+\alpha}}{d_{y}^{-t+k}}\left[d_{y}^{-t+k} \cdot\left|\partial^{\gamma} u_{i}(y)-\partial^{\gamma} u(y)\right|\right] & \\
\leq \frac{d_{x}^{t-s+\alpha}}{|x-y|^{\alpha}}\left\|u-u_{i}\right\|_{k, \beta}^{t}+C & +\frac{1}{|x-y|^{\alpha}} \frac{d_{x}^{-s+k+\alpha}}{d_{y}^{-t+k}}\left\|u-u_{i}\right\|_{k, \beta}^{t} .
\end{aligned}
$$

BULLETIN DE LA SOCIÉTÉ MATHÉMATIQUE DE FRANCE 
Comme $\lim _{i \rightarrow \infty}\left\|u-u_{i}\right\|_{k, \beta}^{t}=0$, on en déduit (à $x, y$ fixés et $i \rightarrow \infty$ ) :

$$
\min \left(d_{x}^{-s+k+\alpha}, d_{y}^{-s+k+\alpha}\right) \frac{\left|\partial^{\gamma} u(x)-\partial^{\gamma} u(y)\right|}{|x-y|^{\alpha}} \leq C
$$

et donc

$$
\sup _{\substack{x, y \in B \\ x \neq y}}\left[\min \left(d_{x}^{-s+k+\alpha}, d_{y}^{-s+k+\alpha}\right) \frac{\left|\partial^{\gamma} u(x)-\partial^{\gamma} u(y)\right|}{|x-y|^{\alpha}}\right] \leq C .
$$

D'où le résultat annoncé, en prenant pour $\mathrm{C}^{\text {te }}(k, n)$ le nombre de multiindices $\gamma$ de longueur au plus $k$ augmenté du nombre de multi-indices $\gamma$ de longueur exactement $k$.

6.3. Construction d'une sur-solution explicite de l'équation $(\Delta+K) \varphi=\rho^{s}$.

Dans cette partie on cherche $\varphi \in \Lambda_{\infty}^{s}$ telle que $(\Delta+K) \varphi \geq \rho^{s}$ où $s$ vérifie la condition :

$$
\begin{cases}K>s(s-(n-1)) & \text { si } s<0 \text { ou } s \geq \frac{1}{2}(n-2) \\ K>-\frac{1}{2} s n & \text { si } 0 \leq s \leq \frac{1}{2}(n-2)\end{cases}
$$

Lemme 8. - Si $\varphi=\Phi \circ \rho$, où $\left.\left.\Phi \in C^{2}(] 0, \frac{1}{2}\right]\right)$ et $\rho(x)=\frac{1}{2}\left(1-|x|^{2}\right)$ alors,

$$
\Delta \varphi=\rho^{2}(2 \rho-1) \Phi^{\prime \prime}(\rho)+\left[(4-n) \rho^{2}+(n-2) \rho\right] \Phi^{\prime}(\rho) .
$$

Preuve. - On a

$$
\Delta \varphi=-H^{i j}\left(\partial_{i} \partial_{j} \varphi-\Gamma_{i j}^{k} \partial_{k} \varphi\right) .
$$

Calculons les coefficients du Laplacien :

$$
\begin{gathered}
H_{i j}=\rho^{-2} \delta_{i j}, \quad \partial_{s} H_{i j}=\frac{2 x_{s}}{\rho^{3}} \delta_{i j} \\
\Gamma_{i j}^{k}=\frac{1}{2} H^{k s}\left(\partial_{i} H_{s j}+\partial_{j} H_{s i}-\partial_{s} H_{i j}\right)=\frac{1}{\rho}\left(x_{i} \delta_{k j}+x_{j} \delta_{k i}-x_{k} \delta_{i j}\right) .
\end{gathered}
$$

Donc

$$
H^{i j} \Gamma_{i j}^{k} \partial_{k} \varphi=\rho\left(2 \sum_{j=1}^{n} x_{j} \partial_{j} \varphi-n \sum_{j=1}^{n} x_{k} \partial_{k} \varphi\right)=\rho(2-n) \sum_{j=1}^{n} x_{j} \partial_{j} \varphi
$$

TOME $125-1997-\mathrm{N}^{\circ} 3$ 
ainsi

$$
\Delta \varphi=-\rho^{2} \sum_{j=1}^{n} \partial_{j}^{2} \varphi+\rho(2-n) \sum_{j=1}^{n} x_{j} \partial_{j} \varphi .
$$

Si $\varphi=\Phi \circ \rho$, alors

$$
\partial_{j} \varphi=-x_{j} \Phi^{\prime}(\rho), \quad \partial_{j}^{2} \varphi=-\Phi^{\prime}(\rho)+x_{j}^{2} \Phi^{\prime \prime}(\rho) .
$$

Ainsi

$$
\begin{aligned}
\Delta \varphi & =\rho^{2}\left[n \Phi^{\prime}(\rho)+(2 \rho-1) \Phi^{\prime \prime}(\rho)\right]+(2-n) \rho(2 \rho-1) \Phi^{\prime}(\rho) \\
& =\rho^{2}(2 \rho-1) \Phi^{\prime \prime}(\rho)+\left[(4-n) \rho^{2}+(n-2) \rho\right] \Phi^{\prime}(\rho) .
\end{aligned}
$$

Proposition 9. - Soit s vérifiant (C) et soit

$$
M(n, s, K):= \begin{cases}\frac{1}{K+s(n-1-s)} & \text { si } s<0 \text { ou } s \geq \frac{1}{2}(n-2) \\ \frac{1}{K+\frac{1}{2} s n} & \text { si } 0 \leq s \leq \frac{1}{2}(n-2)\end{cases}
$$

Si $\varphi=M \rho^{s}$, alors on $a(\Delta+K) \varphi \geq \rho^{s}$.

Preuve. - Calculons

$$
\begin{aligned}
\Delta\left(\rho^{s}\right) & =\rho^{2}(2 \rho-1) s(s-1) \rho^{s-2}+\left[(4-n) \rho^{2}+(n-2) \rho\right] s \rho^{s-1} \\
& =s \rho^{s}[(2 s-n+2) \rho+n-1-s]
\end{aligned}
$$

d'où

$$
(\Delta+K)\left(\rho^{s}\right)=\rho^{s}[s(2 s-n+2) \rho+s(n-1-s)+K] .
$$

- Si $2 s-n+2 \geq 0$ ou $s<0$,

$$
(\Delta+K)\left(\rho^{s}\right) \geq \rho^{s}[s(n-1-s)+K] .
$$

- Si $2 s-n+2 \leq 0$ et $s \geq 0$,

$(\Delta+K)\left(\rho^{s}\right) \geq \rho^{s}\left[s\left(s-\frac{1}{2} n+1\right)+s(n-1-s)+K\right] \geq\left[\frac{1}{2} n s+K\right] \rho^{s}$.

Remarque. - Si $s=\frac{1}{2}(n-2)$, on a :

$$
\frac{1}{2} n s=s(n-1-s)=\frac{4}{n-2} .
$$

BULLETIN DE LA SOCIÉTÉ MATHÉMATIQUE DE FRANCE 
Corollaire 6 . - Si $s \in]-1, n\left[\right.$ et si $\varphi=M(n, s, n) \rho^{s}$, alors

$$
(\Delta+n) \varphi \geq \rho^{s}=\frac{1}{M} \varphi
$$

Preuve. - Avec $K=n$, la condition (C) devient :

$$
\begin{aligned}
& \begin{cases}n>s(s-(n-1)) & \text { si } s<0 \text { ou } s \geq \frac{1}{2}(n-2), \\
n>-\frac{1}{2} s n & \text { si } 0 \leq s \leq \frac{1}{2}(n-2)\end{cases} \\
& \Longleftrightarrow\left\{\begin{array}{l}
\frac{1}{2}(n-2) \leq s<n \text { ou }-1<s<0, \\
0 \leq s \leq \frac{1}{2}(n-2) .
\end{array}\right.
\end{aligned}
$$

L'intervalle total permis pour $s$ est donc bien ] - $1, n[$. Le corollaire suit donc de la proposition précédente.

\section{BIBLIOGRAPHIE}

[A] Aubin (Th). - Nonlinear analysis on manifold. Monge-Ampère Equations. - Springer-Verlag, 1982.

[AO] Aviles (P) and Mcowen (R.). - Conformal deformations of complete manifolds with negative curvature, J. Diff. Geom., t. 21, 1985, p. 269-281.

[BE] Besse (A). - Einstein manifolds. - Springer-Verlag, 1987.

[CCY] Chen (C.Y), Chen (K.S.) and Yu (W.N.). - Conformal Deformations of Metrics on $H^{n}(-1)$ with Prescribed Scalar Curvature, Chinese J. Math., t. 16, n 3 , 1988, p. 157-187.

[GT] Gilbarg (D) and Trudinger (N.S.). - Elliptic Partial Differential Equations of Second Order. - Springer-Verlag, $2^{\text {nd }}$ edition, 1983.

[GL] Graham (C.R) and Lee (J.M.). - Einstein metrics with prescribed infinity on the ball, Adv. in Math., t. 87, $\mathrm{n}^{\circ}$ 2, 1991, p. 186-225.

[L] Leung (M.C). - Pinching theorem on asymptotically hyperbolic spaces, Internat. J. Math., t. 4, $\mathrm{n}^{\circ} 5$, 1993, p. 841-857.

[LTY] Li (P), TAM (L.F.) and YANG (D.G.). - On the elliptic equations $\Delta u+k u-K u^{p}=0$ on complete Riemannian manifolds and their geometric applications, preprint 1994.

TOME $125-1997-\mathrm{N}^{\circ} 3$ 
[MO1] Min-Oo (M). - Scalar curvature rigidity of asymptotically hyperbolic spin manifolds, Math. Ann., t. 285, 1989, p. 527-539.

[MO2] Min-oo (M). - Erratum, en préparation, 1997.

[N] NI (W.-M). - On the elliptic equation $\Delta u+K(x) u^{\frac{n+2}{n-2}}=0$ its generalisation, and application in geometry, Indiana. Univ. Math. J., t. 31, no 4, 1982, p. 493-529.

[RRV] Ratto (A), Rigoli (M.) and Veron(L.). - Scalar curvature and conformal deformations of noncompact Riemannian manifolds, preprint 1995 . 